\title{
CROSSED PRODUCTS BY MINIMAL HOMEOMORPHISMS
}

\author{
HUAXIN LIN AND N. CHRISTOPHER PHILLIPS
}

\begin{abstract}
Let $X$ be an infinite compact metric space with finite covering dimension and let $h: X \rightarrow X$ be a minimal homeomorphism. We show that the associated crossed product $\mathrm{C}^{*}$-algebra $A=C^{*}(\mathbb{Z}, X, h)$ has tracial rank zero whenever the image of $K_{0}(A)$ in $\operatorname{Aff}(T(A))$ is dense. As a consequence, we show that these crossed product $\mathrm{C}^{*}$-algebras are in fact simple AH algebras with real rank zero. When $X$ is connected and $h$ is further assumed to be uniquely ergodic, then the above happens if and only if the rotation number associated to $h$ has irrational values.

By applying the classification result for nuclear simple $\mathrm{C}^{*}$-algebras with tracial rank zero, we show that two such dynamical systems have isomorphic crossed products if and only if they have isomorphic scaled ordered $K$-theory.
\end{abstract}

\section{INTRODUCTION}

Transformation group $\mathrm{C}^{*}$-algebras of minimal homeomorphisms of compact metric spaces have a long history in the theory of $\mathrm{C}^{*}$-algebras. The irrational rotation algebras, among the most prominent examples in the whole subject, have this form. The remarkable work of Giordano, Putnam, and Skau [12] shows that the transformation group $\mathrm{C}^{*}$-algebras of two minimal homeomorphisms of the Cantor set are isomorphic if and only if the homeomorphisms are strong orbit equivalent. The transformation group $\mathrm{C}^{*}$-algebras of Furstenberg transformations on toruses have also attracted considerable attention; as just a few examples, we mention [32, 16, [17, and [42. Connes' example (in Section 5 of [5]) of a simple $\mathrm{C}^{*}$-algebra with no nontrivial projections is the transformation group $\mathrm{C}^{*}$-algebra of a minimal diffeomorphism of $S^{3}$.

One naturally wants to understand the structure of such $\mathrm{C}^{*}$-algebras. That the transformation group $\mathrm{C}^{*}$-algebras of minimal homeomorphisms of the Cantor set are AT algebras (direct limits of circle algebras) with real rank zero is implicit in Section 8 of [13, with the main step having been done in [41. Elliot and Evans proved in 7 that the irrational rotation algebras are AT algebras with real rank zero. In particular, these algebras all belong to the class known currently to be classifiable by K-theoretic invariants in the sense of the Elliott classification program [6]. It is proved in a very long and still unpublished paper 31] (see the survey article [30]) that the transformation group $\mathrm{C}^{*}$-algebra of a minimal diffeomorphism $h$ of a compact smooth manifold $X$ is a direct limit, with no dimension growth, of recursive subhomogeneous $\mathrm{C}^{*}$-algebras. Using this result, one can deduce that

Date: 14 Aug. 2004

2000 Mathematics Subject Classification. Primary 37B05, 46L55; Secondary 37A55, 46L35, $54 \mathrm{H} 20$.

Research of the first author partially supported by NSF grants DMS 009703 and DMS 0355273 , and the Shanghai Priority Academic Disciplines. Research of the second author partially supported by NSF grant DMS 0302401. 
if the $\mathrm{K}$-theory and traces on $C^{*}(\mathbb{Z}, X, h)$ are consistent with this algebra having real rank zero (in the sense described in the next paragraph), then $A$ has tracial rank zero (is tracially AF) in the sense of [20, 21] (see Definition 1.1] below), and is hence classifiable.

In this paper, we prove the following. Let $X$ be an infinite compact metric space with finite covering dimension, and let $h: X \rightarrow X$ be a minimal homeomorphism. Let $A=C^{*}(\mathbb{Z}, X, h)$ be the transformation group $\mathrm{C}^{*}$-algebra. Suppose (notation explained below) that the map $\rho_{A}: K_{0}(A) \rightarrow \operatorname{Aff}(T(A))$ has dense range. (It is well known, and proved in Proposition 1.10(a) of [36], that this condition is necessary for $A$ to have real rank zero.) Then in fact $A$ has tracial rank zero. Here $T(A)$ is the space of tracial states on $A$ with the weak* topology, and if $\Delta$ is a compact convex set then $\operatorname{Aff}(\Delta)$ is the space of real valued affine continuous functions on $\Delta$. The map $\rho_{A}: K_{0}(A) \rightarrow \operatorname{Aff}(T(A))$ is defined by $\rho_{A}(\eta)(\tau)=\tau_{*}(\eta)$ for $\eta \in K_{0}(A)$ and $\tau \in T(A)$.

It follows that $A$ is classifiable, and is in fact a simple unital AH algebra with real rank zero. Furthermore, if the transformation group $\mathrm{C}^{*}$-algebras of two minimal homeomorphisms of finite dimensional compact metric spaces, both satisfying the dense range condition, have isomorphic scaled ordered K-theory, then they are isomorphic. This result gives new examples of distinct minimal homeomorphisms with isomorphic transformation group $\mathrm{C}^{*}$-algebras.

The proof has the advantage over 31] of being short, and of not requiring any smoothness on $X$ or on $h$. It has the disadvantage of giving no information about the transformation group $\mathrm{C}^{*}$-algebra when $\rho_{C^{*}(\mathbb{Z}, X, h)}$ does not have dense range. The direct limit decomposition in 31] requires no such assumption, and implies that the transformation group $\mathrm{C}^{*}$-algebra has stable rank one and that the order on projections is determined by traces even when it does not have real rank zero, such as for the example of Section 5 of [5].

To describe the main idea of the proof, let $u \in C^{*}(\mathbb{Z}, X, h)$ be the canonical unitary, and let $y \in X$. Consider the subalgebra $B=C^{*}(\mathbb{Z}, X, h)_{\{y\}}$ of $C^{*}(\mathbb{Z}, X, h)$ generated by $C(X)$ and all uf with $f \in C(X)$ and $f(y)=0$. (This subalgebra plays a key role in [29, and is a generalization of a subalgebra originally introduced in [40].) Under our hypotheses, $\rho_{B}: K_{0}(B) \rightarrow \operatorname{Aff}(T(B))$ also has dense range. If there are only countably many ergodic $h$-invariant measures, then Theorem 4.4 of 36 implies that $B$ has tracial rank zero. Even if this is not the case, one can combine intermediate results from [36] and dynamical arguments to show that $B$ has tracial rank zero for "most" (presumably actually all) choices of $y$. Having this, one can use Berg's technique to find arbitrarily "small" approximately $h$-invariant (in a suitable sense) projections $p$ in $C^{*}(\mathbb{Z}, X, h)$ and functions $f \in C(X)$ such that $f=1$ on a neighborhood of $y$ and such that $p f=f$. The corners $(1-p) C^{*}(\mathbb{Z}, X, h)(1-p)$ are, in a suitable sense, approximately contained in $B$. Since $B$ has tracial rank zero and $1-p$ is "large", one can then deduce that $C^{*}(\mathbb{Z}, X, h)$ has tracial rank zero.

This paper is organized as follows. In Section 2 we introduce notation and give some elementary properties of algebras related to the subalgebra $B$ in the description above. In Section 3, we prove that, under our hypotheses, $B$ (usually) has tracial rank zero. Section 4 contains the proof that $C^{*}(\mathbb{Z}, X, h)$ has tracial rank zero. In Section 5 we use our result to examine some examples. In particular, we prove isomorphism of the transformation group $\mathrm{C}^{*}$-algebras in some pairs of 
examples in 33. for which the isomorphism question was left open there, due to lack of smoothness. Section [ 6 considers approximate conjugacy of the homeomorphisms in some of these examples.

We use the notation $p \precsim q$ to mean that a projection $p$ is Murray-von Neumann equivalent to a subprojection of a projection $q$. By convention, if $B$ is said to be a unital subalgebra of a unital $\mathrm{C}^{*}$-algebra $A$, then, unless otherwise specified, we mean that the identity of $B$ is the same as that of $A$.

For the convenience of the reader, we recall the meaning of tracial rank zero (or tracial topological rank zero) for simple $\mathrm{C}^{*}$-algebras.

Definition 1.1. Let $A$ be a simple unital $\mathrm{C}^{*}$-algebra. Then $A$ has tracial rank zero if for every finite subset $\mathcal{F} \subset A$, every $\varepsilon>0$, and every nonzero positive element $c \in A$, there exists a projection $p \in A$ and a unital finite dimensional subalgebra $E \subset p A p$ such that:

(1) $\|[a, p]\|<\varepsilon$ for all $a \in \mathcal{F}$.

(2) $\operatorname{dist}($ pap,$E)<\varepsilon$ for all $a \in \mathcal{F}$.

(3) $p$ is Murray-von Neumann equivalent to a projection in $\overline{c A c}$.

That this definition is equivalent to the original one follows from Proposition 3.8 of 20 .

Acknowledgements: The second author would like to thank Mike Boyle and Tomasz Downarowicz for valuable email correspondence on minimal homeomorphisms of finite dimensional compact metric spaces. In particular, Mike Boyle called attention to the reference [18].

\section{Subalgebras of the transformation group $\mathrm{C}^{*}$-Algebra}

Let $X$ be a compact metric space, and let $h: X \rightarrow X$ be a homeomorphism. Then the induced automorphism $\alpha$ of $C(X)$ is $\alpha(f)=f \circ h^{-1}$. In the transformation group $\mathrm{C}^{*}$-algebra $C^{*}(\mathbb{Z}, X, h)$, we normally write $u$ for the standard unitary representing the generator of $\mathbb{Z}$. Then $u f u^{*}=\alpha(f)=f \circ h^{-1}$.

Notation 2.1. Let $X$ be a compact metric space, and let $h: X \rightarrow X$ be a homeomorphism. For a nonempty closed subset $Y \subset X$, we define the $\mathrm{C}^{*}$-subalgebra $C^{*}(\mathbb{Z}, X, h)_{Y}$ to be

$$
C^{*}(\mathbb{Z}, X, h)_{Y}=C^{*}\left(C(X), u C_{0}(X \backslash Y)\right) \subset C^{*}(\mathbb{Z}, X, h) .
$$

We will often let $A$ denote the transformation group $\mathrm{C}^{*}$-algebra $C^{*}(\mathbb{Z}, X, h)$, in which case we refer to $A_{Y}$.

The case of direct use to us is $Y=\{y\}$. The following immediate structural fact will be crucial.

Remark 2.2. Let the notation be as in Notation 2.1] with $A=C^{*}(\mathbb{Z}, X, h)$. If $Y_{1} \supset Y_{2} \supset \cdots$ is a decreasing sequence of closed subsets of $X$ with $\bigcap_{n=1}^{\infty} Y_{n}=\{y\}$, then $A_{\{y\}}=\underline{\lim } A_{Y_{n}}$.

We need the following description of $C^{*}(\mathbb{Z}, X, h)_{Y}$ when $h$ is minimal and $\operatorname{int}(Y) \neq$ $\varnothing$. These results are originally from the unpublished preprint 28. Outlines of the proofs can be found in Section 3 of the survey article [29; details (of this and much more) will appear in 31 . 
The first theorem gives the decomposition of $X$ into Rokhlin towers associated to $Y$ and the first return times to $Y$. The bases of the towers are taken to be subsets of $h(Y)$ rather than, as would be more usual, subsets of $Y$. This is the convenient choice for use with our definition of $C^{*}(\mathbb{Z}, X, h)_{Y}$. For recursive subhomogeneous algebras and recursive subhomogeneous decompositions as in the second theorem, see Section 1 of [34].

Theorem 2.3. Let $X$ be an infinite compact metric space, and let $h: X \rightarrow X$ be a minimal homeomorphism. Let $Y \subset X$ be closed and have nonempty interior. For $y \in Y$ set $r(y)=\min \left\{n \geq 1: h^{n}(y) \in Y\right\}$. Then $\sup _{y \in Y} r(y)<\infty$. Let $n(0)<n(1)<\cdots<n(l)$ be the distinct values in the range of $r$, and for $0 \leq k \leq l$ set

$$
Y_{k}=\overline{\{y \in Y: r(y)=n(k)\}} \quad \text { and } \quad Y_{k}^{\circ}=\operatorname{int}(\{y \in Y: r(y)=n(k)\}) .
$$

Then:

(1) The sets $h^{j}\left(Y_{k}^{\circ}\right)$, for $0 \leq k \leq l$ and $1 \leq j \leq n(k)$, are disjoint.

(2) $\bigcup_{k=0}^{l} \bigcup_{j=1}^{n(k)} h^{j}\left(Y_{k}\right)=X$.

(3) $\bigcup_{k=0}^{l} h^{n(k)}\left(Y_{k}\right)=Y$.

Theorem 2.4. Let $X, h, Y$, and the other notation be as in Notation 2.1 and Theorem 2.3] and let $A=C^{*}(\mathbb{Z}, X, h)$. Set $B_{Y}=\bigoplus_{k=0}^{l} C\left(Y_{k}, M_{n(k)}\right)$. Then $A_{Y}$ has a recursive subhomogeneous decomposition with base spaces $Y_{0}, Y_{1}, \ldots, Y_{l}$ and with standard representation $\sigma: A_{Y} \rightarrow B_{Y}$, such that:

(1) If $f \in C(X) \subset A_{Y}$ then $\sigma(f)_{k} \in C\left(Y_{k}, M_{n(k)}\right)$ is given by

$$
\sigma(f)_{k}=\operatorname{diag}\left(\left(\left.f\right|_{h\left(Y_{k}\right)}\right) \circ h,\left(\left.f\right|_{h^{2}\left(Y_{k}\right)}\right) \circ h^{2}, \ldots,\left(\left.f\right|_{h^{n(k)}\left(Y_{k}\right)}\right) \circ h^{n(k)}\right) .
$$

(2) If $f \in C_{0}(X \backslash Y)$ then $\sigma(u f)_{k} \in C\left(Y_{k}, M_{n(k)}\right)$ is given by

$$
\sigma_{k}(u f)=\left(\begin{array}{ccccc}
0 & 0 & \cdots & 0 & 0 \\
1 & 0 & \cdots & 0 & 0 \\
0 & 1 & \cdots & 0 & 0 \\
\vdots & \vdots & \ddots & \vdots & \vdots \\
0 & 0 & \cdots & 1 & 0
\end{array}\right) \sigma_{k}(f)
$$

The following result is due to Qing Lin (Proposition 12 of 28). Since that paper has not been published, we give a proof here.

Proposition 2.5. Let $X$ be an infinite compact metric space, and let $h: X \rightarrow X$ be a minimal homeomorphism. Let $A=C^{*}(\mathbb{Z}, X, h)$, and adopt Notation 2.1] Let $y \in X$. Then $A_{\{y\}}$ is simple.

Proof. Let $I \subset A_{\{y\}}$ be a nonzero ideal. Then $I \cap C(X)$ is an ideal in $C(X)$, so we can write $I \cap C(X)=C_{0}(U)$ for some open set $U$, which is necessarily given by

$$
U=\{x \in X \text { : there is } f \in I \cap C(X) \text { such that } f(x) \neq 0\} .
$$

We first claim that $U \neq \varnothing$. Write $A_{\{y\}}=\underset{\lim }{\rightarrow} A_{Y_{m}}$ as in Remark 2.2 with $Y_{m}$ chosen so that $\operatorname{int}\left(Y_{m}\right) \neq \varnothing$. Then there exists $m$ such that $A_{Y_{m}} \cap I \neq\{0\}$. Let $a$ be a nonzero element of this intersection. Using Theorem 2.4 one can fairly easily prove that there is $N$ such that every element of $A_{Y_{m}}$ can be written in the form $\sum_{n=-N}^{N} f_{n} u^{n}$ with $f_{n} \in C(X)$ for $-N \leq n \leq N$. Moreover, if $a \neq 0$ and one writes $a^{*} a=\sum_{n=-N}^{N} f_{n} u^{n}$, then $f_{0} \neq 0$. Choose $x \in X$ such that $f_{0}(x) \neq 0$, choose a 
neighborhood $V$ of $x$ such that the sets $h^{n}(V)$, for $-N \leq n \leq N$, are disjoint, and choose $g \in C(X)$ such that $\operatorname{supp}(g) \subset V$ and $g(x) \neq 0$. Then $g \in A_{\{y\}}$, and one checks that

$$
g a^{*} a g=\sum_{n=-N}^{N} g f_{n} u^{n} g=\sum_{n=-N}^{N} g\left(g \circ h^{n}\right) f_{n} u^{n}=g^{2} f .
$$

So $g^{2} f$ is a nonzero element of $I \cap C(X)$, proving the claim.

We next claim that $h^{-1}(U \backslash\{h(y)\}) \subset U$. So let $x \in U \backslash\{h(y)\}$. Choose $f \in$ $I \cap C(X)$ such that $f(x) \neq 0$, and choose $g \in C_{0}(X \backslash\{y\})$ such that $g\left(h^{-1}(x)\right) \neq 0$. Then $u g \in A_{\{y\}}$, and

$$
(u g)^{*} f(u g)=\bar{g} u^{*} f u g=|g|^{2}(f \circ h) .
$$

Thus $|g|^{2}(f \circ h) \in I \cap C(X)$ and is nonzero at $h^{-1}(x)$. This proves the claim.

We further claim that $h(U \backslash\{y\}) \subset U$. The proof is similar: let $x \in U \backslash\{y\}$, let $f \in I \cap C(X)$ and $g \in C_{0}(X \backslash\{y\})$ be nonzero at $x$, and consider $(u g) f(u g)^{*}$.

Now set $Z=X \backslash U$. The last two claims above imply that if $x \in X$ and $x$ is not in the orbit of $y$, then $h^{k}(x) \in Z$ for all $k \in \mathbb{Z}$. Since $h$ is minimal, $Z$ is closed, and $Z \neq X$, this is impossible. If $h^{n}(y) \in Z$ for some $n>0$, then $h^{-1}(U \backslash\{h(y)\}) \subset U \backslash\{h(y)\}$ implies $h^{k}(y) \in Z$ for all $k \geq n$. Since $\left\{h^{k}(y): k \geq n\right\}$ is dense by minimality, this is also a contradiction. Similarly, if $h^{n}(y) \in Z$ for some $n \leq 0$, then $Z$ would contain the dense set $\left\{h^{k}(y): k \leq n\right\}$, again a contradiction. So $Z=\varnothing$, whence $U=X$, and $1 \in I$.

For convenience, we also reproduce the following result of Qing Lin (Proposition 16 of [28]). The proof is sketched in the proof of Theorem 1.2 of [29].

Proposition 2.6. Let $X$ be an infinite compact metric space, and let $h: X \rightarrow X$ be a minimal homeomorphism. Let $A=C^{*}(\mathbb{Z}, X, h)$, and adopt Notation 2.1 Let $y \in X$. Then the restriction map $T(A) \rightarrow T\left(A_{\{y\}}\right)$ is bijective.

\section{The tracial Rank of $A_{\{y\}}$}

Let the notation be as in the previous section, with $A=C^{*}(\mathbb{Z}, X, h)$. We prove in this section that if the space $X$ is finite dimensional, $h$ is minimal, and the image of $K_{0}(A)$ in $\operatorname{Aff}(T(A))$ is dense, then $A_{\{y\}}$ has tracial rank zero for "most" $y \in X$. The result is surely true for all $y \in X$, and the proof should not be much harder, but this version is more convenient to prove and suffices for our purposes.

If $A$ has at most countably many extreme tracial states, equivalently, if $h$ has at most countably many ergodic probability measures, then this result (for all $y$ ) is easily obtained from Theorem 4.4 of [36]. The point of the argument here is that it gives the result when $T(A)$ is completely arbitrary.

Definition 3.1. Let $X$ be a compact metric space, and let $h: X \rightarrow X$ be a homeomorphism. A Borel set $Z \subset X$ is called universally null if $\mu(Z)=0$ for every $h$-invariant Borel probability measure $\mu$ on $X$.

Proposition 3.2. Let $X$ be an infinite compact metric space with finite covering dimension, and let $h: X \rightarrow X$ be a homeomorphism whose set of periodic points has dimension zero or is empty. For every $\varepsilon>0$ there are disjoint open sets $U_{1}, U_{2}, \ldots, U_{m} \subset X$ such that $\operatorname{diam}\left(U_{j}\right)<\varepsilon$ and $\operatorname{int}\left(\overline{U_{j}}\right)=U_{j}$ for $1 \leq j \leq m$, and such that $X \backslash \bigcup_{j=1}^{m} U_{j}$ is universally null. 
Proof. Let $d=\operatorname{dim}(X)$. We apply Lemma 3.7 of [18]. For the terminology used there, see Section 1 of the paper, and note that $\omega=\mathbb{N}$. Taking $i$ there to be greater than $1 / \varepsilon$, we obtain closed subsets $T_{1}, T_{2}, \ldots, T_{n} \subset X$ which cover $X$, whose interiors $U_{1}, U_{2}, \ldots, U_{m}$ are disjoint and satisfy $\overline{U_{j}}=T_{j}$, and such that whenever

$$
(j(0), n(0)),(j(1), n(1)), \ldots,(j(d), n(d)) \in\{1,2, \ldots, m\} \times \mathbb{Z}
$$

are $d+1$ distinct pairs, then

$$
h^{n(0)}\left(\partial T_{j(0)}\right) \cap h^{n(1)}\left(\partial T_{j(1)}\right) \cap \cdots \cap h^{n(d)}\left(\partial T_{j(d)}\right)=\varnothing .
$$

Set

$$
Z=X \backslash \bigcup_{j=1}^{m} U_{j} \subset \bigcup_{j=1}^{m} \partial T_{j} .
$$

Then $f=\sum_{n \in \mathbb{Z}} \chi_{h^{n}(Z)}$ is a nonnegative Borel function on $X$ which is bounded by $d$. If $\mu$ is any $h$-invariant Borel probability measure on $X$, then

$$
\sum_{n \in \mathbb{Z}} \mu\left(h^{n}(Z)\right)=\int_{X} f d \mu \leq d .
$$

Since $\mu\left(h^{n}(Z)\right)=\mu(Z)$ for all $n$, it follows that $\mu(Z)=0$.

Corollary 3.3. Let $X$ be an infinite compact metric space with finite covering dimension, and let $h: X \rightarrow X$ be a minimal homeomorphism. Then there exists a dense $G_{\delta}$-set $S \subset X$ such that for every $y \in S$ and every open set $V$ containing $X$, there is a closed set $Y$ such that $y \in \operatorname{int}(Y) \subset Y \subset V$ and $\partial Y$ is universally null.

Proof. Apply Proposition 3.2 with $\varepsilon=\frac{1}{n}$, and let $W_{n}$ be the union of the resulting finite collection of open sets $U_{n, 1}, U_{n, 2}, \ldots, U_{n, m(n)}$. Then $W_{n}$ is dense, since by minimality every $h$-invariant Borel probability measure is nonzero on every nonempty open subset of $X$. So $S=\bigcap_{n=1}^{\infty} W_{n}$ is a dense $G_{\delta}$-set in $X$. Given $y \in S$ and an open set $V$ containing $y$, for $n$ large enough we take $Y=\overline{U_{n, j}}$ with $j$ chosen such that $y \in U_{n, j}$. Note that $\partial Y \subset X \backslash W_{n}$.

Corollary 3.4. Let $X$ be an infinite compact metric space with finite covering dimension, and let $h: X \rightarrow X$ be a minimal homeomorphism. Let $Y \subset X$ be a closed subset with nonempty interior such that $\partial Y$ is universally null, and adopt the notation of Theorem 2.3 Then for $0 \leq k \leq l$ there are disjoint open subsets $U_{k, 1}, U_{k, 2}, \ldots, U_{k, r(k)}$ of $X$ which are contained in $Y_{l}^{\circ}$, such that

$$
X \backslash \bigcup_{k=0}^{l} \bigcup_{j=1}^{n(k)} \bigcup_{i=1}^{r(k)} h^{j}\left(U_{k, i}\right)
$$

is universally null, such that $\operatorname{diam}\left(h^{j}\left(U_{k, i}\right)\right)<\varepsilon$ for $0 \leq k \leq l, 1 \leq j \leq n(k)$, and $1 \leq i \leq r(k)$, and such that the sets $h^{j}\left(U_{k, i}\right)$, for these $j, k, i$, are disjoint.

Proof. Choose $\delta>0$ so small that if $d(x, y)<\delta$ then

$$
d(x, y)<\varepsilon, \quad d(h(x), h(y))<\varepsilon, \quad \ldots, \quad d\left(h^{n(l)}(x), h^{n(l)}(y)\right)<\varepsilon .
$$

Apply Proposition 3.2 with $\delta$ in place of $\varepsilon$. Then take $U_{k, 1}, U_{k, 2}, \ldots, U_{k, r(k)}$ to be the nonempty sets among $U_{1} \cap Y_{k}^{\circ}, U_{2} \cap Y_{k}^{\circ}, \ldots, U_{m} \cap Y_{k}^{\circ}$. Note that $\partial\left(U_{i} \cap Y_{k}^{\circ}\right) \subset$ $\partial U_{i} \cup \partial Y_{k}^{\circ}$. Disjointness follows from Theorem 2.3 (1).

The following lemma is taken from the proof of Lemma 3 of 19. (It has also appeared in other places.) 
Lemma 3.5. Let $A$ be a simple unital $\mathrm{C}^{*}$-algebra, and let $B \subset A$ be a separable commutative unital $\mathrm{C}^{*}$-subalgebra, which we identify with $C(X)$ for some compact metric space $X$. For any $\tau \in T(A)$ let $\mu_{\tau}$ be the Borel probability measure on $X$ induced by $\tau$. Let $Z \subset X$ be a closed subset, and suppose that $\mu_{\tau}(Z)=0$ for all $\tau \in T(A)$. Then for any $\varepsilon>0$ there is an open subset $U \subset X$ containing $Z$ such that $\mu_{\tau}(U)<\varepsilon$ for all $\tau \in T(A)$.

Proof. Choose open sets $U_{1} \supset \bar{U}_{2} \supset U_{2} \supset \cdots \supset Z$ such that $\bigcap_{n=0}^{\infty} U_{n}=Z$. Choose continuous functions $f_{n}: X \rightarrow[0,1]$ such that $\operatorname{supp}\left(f_{n}\right) \subset U_{n}$ and $f_{n}=1$ on $U_{n+1}$. Then $f_{1} \geq f_{2} \geq \cdots$. Each $f_{n}$ defines a function $g_{n} \in \operatorname{Aff}(T(A))$ by $g_{n}(\tau)=\tau\left(f_{n}\right)$. Since $\mu_{\tau}(Z)=0$ for all $\tau \in T(A)$, we have $g_{n}(\tau) \rightarrow 0$ for each $\tau \in T(A)$ by the Dominated Convergence Theorem. Since 0 is continuous on the compact set $T(A)$, Dini's Theorem implies that $g_{n} \rightarrow 0$ uniformly on $T(A)$. Thus, for any $\varepsilon>0$ there exists $n$ such that $\tau\left(f_{n}\right)<\varepsilon$ for all $\tau \in T(A)$. It follows that $\mu_{\tau}\left(U_{n}\right)<\varepsilon$ for all $\tau \in A$.

Corollary 3.6. Let $X$ be a compact metric space, and let $h: X \rightarrow X$ be a minimal homeomorphism. Let $Z \subset X$ be a universally null closed set, and let $\varepsilon>0$. Then there exists an open set $U \subset X$ containing $Z$ such that $\mu(U)<\varepsilon$ for every $h$ invariant Borel probability measure $\mu$ on $X$.

Proof. Apply Lemma 3.5 with $A=C^{*}(\mathbb{Z}, X, h)$ and with $B$ being the canonically embedded copy of $C(X)$. The result follows from the one to one correspondence between $h$-invariant Borel probability measures on $X$ and tracial states on $C^{*}(\mathbb{Z}, X, h)$.

Theorem 3.7. Let $X$ be an infinite compact metric space with finite covering dimension, and let $h: X \rightarrow X$ be a minimal homeomorphism. Let $A=C^{*}(\mathbb{Z}, X, h)$, and adopt Notation 2.1 Suppose that the image of $K_{0}(A)$ in $\operatorname{Aff}(T(A))$ is dense. Then there is a dense $G_{\delta}$-set $S \subset X$ such that for every $y \in S$, the subalgebra $A_{\{y\}}$ has tracial rank zero.

Proof. We begin with the following observations (justified below), which hold for every $y \in X$ :

(1) $A_{\{y\}}$ is simple.

(2) $A_{\{y\}}$ has stable rank one.

(3) The order on projections in $A_{\{y\}}$ is determined by traces, that is, if $p, q \in$ $A_{\{y\}}$ are projections such that $\tau(p)<\tau(q)$ for every tracial state $\tau$, then $p \precsim q$.

(4) The image of $K_{0}\left(A_{\{y\}}\right)$ in $\operatorname{Aff}\left(T\left(A_{\{y\}}\right)\right)$ is dense.

Statement (1) is Proposition 2.5] Statement (2) is Theorem 4.2(1) of [35], and (3) is Theorem 4.2(3) of [35]. For (4), we observe that the restriction map $T(A) \rightarrow$ $T\left(A_{\{y\}}\right)$ is bijective (Proposition [2.6), and that $K_{0}\left(A_{\{y\}}\right) \rightarrow K_{0}(A)$ is a group isomorphism (Theorem 4.1(3) of [35). (The map $K_{0}\left(A_{\{y\}}\right) \rightarrow K_{0}(A)$ is an order isomorphism, but we do not need this fact.) Then (4) follows from the hypothesis that the image of $K_{0}(A)$ in $\operatorname{Aff}(T(A))$ is dense.

Now let $S \subset X$ be as in Corollary 3.3 and let $y \in S$. Given the properties above, we will prove that $A_{\{y\}}$ has tracial rank zero by verifying the following condition: For every finite subset $\mathcal{F} \subset A$ and every $\varepsilon>0$, there exists a projection $p \in A$ and a unital finite dimensional subalgebra $E \subset p A p$ such that:

(5) $\|[a, p]\|<\varepsilon$ for all $a \in \mathcal{F}$. 
(6) For all $a \in \mathcal{F}$ there exists $b \in E$ such that $\|p a p-b\|<\varepsilon$.

(7) $\tau(1-p)<\varepsilon$ for all tracial states $\tau$ on $A$.

That this suffices is Corollary 6.15 of [21] the Fundamental Comparison Property there (stated in Theorem 6.8 of 21]) is just (3) above.

In fact, we need only let the finite set $\mathcal{F}$ run through a cofinal collection of finite subsets of a set $\mathcal{G}$ which generates $A$ as a $\mathrm{C}^{*}$-algebra. Set

$$
\mathcal{G}_{0}=\{f \in C(X): f \text { vanishes on a neighborhood of } y\} .
$$

Then take $\mathcal{G}=\{1\} \cup \mathcal{G}_{0} \cup u \mathcal{G}_{0}$. Our finite sets will have the form $\mathcal{F}=\{1\} \cup \mathcal{F}_{0} \cup u \mathcal{F}_{0}$ for a finite set $\mathcal{F}_{0} \subset \mathcal{G}_{0}$.

Use the hypothesis $y \in S$ to choose closed sets $Z_{1} \supset Z_{2} \supset \cdots$ such that $\bigcap_{m=1}^{\infty} Z_{m}=\{y\}$, such that $y \in \operatorname{int}\left(Z_{m}\right)$ for all $m$, and such that $\partial Z_{m}$ is universally null for all $m$. Then $A_{\{y\}}=\lim A_{Z_{m}}$ by Remark 2.2 Given $\mathcal{F}_{0} \subset \mathcal{G}_{0}$ finite, choose $m_{0}$ so large that $\operatorname{supp}(f) \subset \vec{X} Z_{m_{0}}$ for all $f \in \mathcal{F}_{0}$. Set $Y=Z_{m_{0}}$, and let the notation be as in Theorems 2.3 and 2.4 Choose $\delta>0$ so small that whenever $x_{1}, x_{2} \in X$ satisfy $d\left(x_{1}, x_{2}\right)<\delta$ then $\left|f\left(x_{1}\right)-f\left(x_{2}\right)\right|<\frac{1}{3} \varepsilon$ for all $f \in \mathcal{F}$. Use Corollary 3.4 to find, for $0 \leq k \leq l$, disjoint open subsets $U_{k, 1}, U_{k, 2}, \ldots, U_{k, r(k)}$ of $X$ which are contained in $Y_{l}^{\circ}$, such that, with

$$
U=\bigcup_{k=0}^{l} \bigcup_{j=1}^{n(k)} \bigcup_{i=1}^{r(k)} h^{j}\left(U_{k, i}\right)
$$

the set $X \backslash U$ is universally null, and such that $\operatorname{diam}\left(h^{j}\left(U_{k, i}\right)\right)<\delta$ for $0 \leq k \leq l$, $1 \leq j \leq n(k)$, and $1 \leq i \leq r(k)$. By Corollary 3.6 there is an open set $V_{0} \subset X$ such that $X \backslash U \subset V_{0}$ and $\mu\left(V_{0}\right)<\varepsilon$ for every $h$-invariant Borel probability measure $\mu$ on $X$. Choose an open set $V$ with $X \backslash U \subset V \subset \bar{V} \subset V_{0}$. Set $W_{k, i}=(X \backslash \bar{V}) \cap U_{k, i}$ for $0 \leq k \leq l$ and $1 \leq i \leq r(k)$. Choose continuous functions

$$
g_{k, i}^{(0)}, g_{k, i}^{(1)}, g_{k, i}^{(2)}: X \rightarrow[0,1]
$$

such that

$$
\begin{gathered}
\operatorname{supp}\left(g_{k, i}^{(2)}\right) \subset h\left(U_{k, i}\right), \quad g_{k, i}^{(2)} g_{k, i}^{(1)}=g_{k, i}^{(1)}, \\
g_{k, i}^{(1)} g_{k, i}^{(0)}=g_{k, i}^{(0)}, \quad \text { and } \quad g_{k, i}^{(0)} \chi_{h\left(W_{k, i}\right)}=\chi_{h\left(W_{k, i}\right)}
\end{gathered}
$$

for $0 \leq k \leq l$ and $1 \leq i \leq r(k)$.

Because $A_{\{y\}}=\lim _{\longrightarrow} A_{Z_{m}}$ and using (4), Proposition 3.5 of [36 provides projections $q_{k, i} \in A_{\{y\}}$ (actually, in $A_{Z_{m}}$ for some $m \geq m_{0}$ ) such that

$$
g_{k, i}^{(2)} q_{k, i}=q_{k, i} \quad \text { and } \quad q_{k, i} g_{k, i}^{(0)}=g_{k, i}^{(0)}
$$

for $0 \leq k \leq l$ and $1 \leq i \leq r(k)$. Then define $p_{k, i, j}=u^{j-1} q_{k, i} u^{-(j-1)}$ (so that $\left.p_{k, i, 1}=q_{k, i}\right)$, and set

$$
p_{k, i}=\sum_{j=1}^{n(k)} p_{k, i, j} \quad \text { and } \quad E_{k, i}=\operatorname{span}\left(\left\{u^{j_{1}-1} q_{k, i} u^{j_{2}-1}: 1 \leq j_{1}, j_{2} \leq n(k)\right\}\right) .
$$

It is now convenient to introduce the index sets

$$
Q=\{(k, i, j): 0 \leq k \leq l, 1 \leq i \leq r(k), \text { and } 1 \leq j \leq n(k)\}
$$

and

$$
Q_{0}=\{(k, i, j): 0 \leq k \leq l, 1 \leq i \leq r(k), \text { and } 1 \leq j \leq n(k)-1\} .
$$


Thus, using Theorem 2.3 (3) for the second equation,

$$
U=\bigcup_{(k, i, j) \in Q} h^{j}\left(U_{k, i}\right) \text { and } U \cap(X \backslash Y)=\bigcup_{(k, i, j) \in Q_{0}} h^{j}\left(U_{k, i}\right) .
$$

We claim that (proofs below):

(8) $p_{k, i} \in A_{\{y\}}$ and $E_{k, i} \subset A_{\{y\}}$.

(9) $\left(g_{k, i}^{(2)} \circ h^{-(j-1)}\right) p_{k, i, j}=p_{k, i, j}$ and $p_{k, i, j}\left(g_{k, i}^{(0)} \circ h^{-(j-1)}\right)=g_{k, i}^{(0)} \circ h^{-(j-1)}$ for $(k, i, j) \in Q$.

(10) The $p_{k, i, j}$, for $(k, i, j) \in Q$, are orthogonal projections.

(11) The $p_{k, i}$, for $0 \leq k \leq l$ and $1 \leq i \leq r(k)$, are orthogonal projections.

(12) $E_{k, i}$ is a finite dimensional $\mathrm{C}^{*}$-algebra with identity $p_{k, i}$ (which is isomorphic to $M_{n(k)}$, and has matrix units $\left.e_{j_{1}, j_{2}}=u^{j_{1}-1} q_{k, i} u^{j_{2}-1}\right)$.

Then we set

$$
p=\sum_{k=0}^{l} \sum_{i=1}^{r(k)} p_{k, i} \quad \text { and } \quad E=\bigoplus_{k=0}^{l} \bigoplus_{i=1}^{r(k)} p_{k, i} .
$$

It follows from the claims above that:

(13) $p$ is a projection in $A_{\{y\}}$.

(14) $E$ is a finite dimensional C*-subalgebra of $A_{\{y\}}$ with identity $p$.

We further claim that (again, proofs below):

(15) $\|[a, p]\|<\varepsilon$ for all $a \in \mathcal{F}$.

(16) For all $a \in \mathcal{F}$ there exists $b \in E$ such that $\|p a p-b\|<\varepsilon$.

(17) $\tau(1-p)<\varepsilon$ for all tracial states $\tau$ on $A_{\{y\}}$.

Statements (13) through (17) yield the condition for tracial rank zero given above, so the proof will be complete once (8) through (12) and (15) through (17) are verified.

We begin with (8). It is enough to prove that $u^{j-1} q_{k, i} \in A_{\{y\}}$ for $1 \leq j \leq n(k)$. We write

$$
u^{j-1} q_{k, i}=u^{j-1}\left(g_{k, i}^{(2)}\right)^{j-1} q_{k, i}=u\left(g_{k, i}^{(2)} \circ h^{-(j-2)}\right) \cdots u\left(g_{k, i}^{(2)} \circ h^{-1}\right) \cdot u g_{k, i}^{(2)} \cdot q_{k, i} .
$$

The functions $g_{k, i}^{(2)}, g_{k, i}^{(2)} \circ h^{-1}, \ldots, g_{k, i}^{(2)} \circ h^{-(j-2)}$ are supported in

$$
h\left(U_{k, i}\right) \subset h\left(Y_{k}\right), h^{2}\left(U_{k, i}\right) \subset h^{2}\left(Y_{k}\right), \ldots, h^{j-1}\left(U_{k, i}\right) \subset h^{j-1}\left(Y_{k}\right),
$$

so that every factor except $q_{k, i}$ in the last expression is in $u C_{0}(X \backslash Y)$. This proves (8).

The relations in (9) are obtained by conjugating the relations

$$
g_{k, i}^{(2)} q_{k, i}=q_{k, i} \quad \text { and } \quad q_{k, i} g_{k, i}^{(0)}=g_{k, i}^{(0)}
$$

by $u^{j-1}$ and using $u^{(j-1)} g u^{-(j-1)}=g \circ h^{-(j-1)}$.

Part (10) follows from (9) together with the observation that $\operatorname{supp}\left(g_{k, i}^{(2)} \circ h^{-(j-1)}\right) \subset$ $h^{j}\left(U_{k, i}\right)$ and the fact that the sets $h^{j}\left(U_{k, i}\right)$, for $(k, i, j) \in Q$, are disjoint. Part (11) is now immediate. In (12), the matrix unit relations are easy once one has the orthogonality relations in (10), and that $E_{k, i}$ is a finite dimensional $\mathrm{C}^{*}$-algebra with identity $p_{k, i}$ is then immediate.

We now prove (15) and (16). By construction, for $(k, i, j) \in Q$ there exists $\lambda_{k, i, j} \in \mathbb{C}$ such that $\left|\lambda_{k, i, j}-f(x)\right|<\frac{1}{3} \varepsilon$ for every $f \in \mathcal{F}_{0}$ and every $x \in h^{j}\left(U_{k, i}\right)$. 
Since $f$ vanishes on $X \backslash Y$, we may require $\lambda_{k, i, j}=0$ when $(k, i, j) \notin Q_{0}$, that is, when $j=n(k)$. Set

$$
b=\sum_{(k, i, j) \in Q} \lambda_{k, i, j} p_{k, i, j},
$$

which is in $E$.

We have

$$
\left\|f \cdot \sum_{(k, i, j) \in Q} g_{k, i}^{(2)} \circ h^{-(j-1)}-\sum_{(k, i, j) \in Q} \lambda_{k, i, j} \cdot\left(g_{k, i}^{(2)} \circ h^{-(j-1)}\right)\right\| \leq \frac{\varepsilon}{3} .
$$

Multiplying on the right by

$$
p=\sum_{(k, i, j) \in Q} p_{k, i, j}
$$

using (9) and disjointness of the supports of the $g_{k, i}^{(2)} \circ h^{-(j-1)}$ for $(k, i, j) \in Q$, we get $\|f p-b\| \leq \frac{1}{3} \varepsilon$. Similarly $\|p f-b\| \leq \frac{1}{3} \varepsilon$ and $\|p f p-b\| \leq \frac{1}{3} \varepsilon<\varepsilon$. Thus $\|[p, f]\| \leq \frac{2}{3} \varepsilon<\varepsilon$. This gives (15) and (16) for $a \in \mathcal{F}_{0}$.

Next, set

$$
e=\sum_{(k, i, j) \in Q_{0}} p_{k, i, j} \leq p .
$$

Because $\lambda_{k, i, j}=0$ when $j=n(k)$, we get $e b=b e=b$, and also

$$
u b=\sum_{(k, i, j) \in Q_{0}} \lambda_{k, i, j} \cdot u p_{k, i, j}=\sum_{(k, i, j) \in Q_{0}} \lambda_{k, i, j} \cdot u^{j} q_{k, i} u^{-(j-1)} \in E .
$$

The next step is to show that $p u f=u e f$. Now

$$
u^{*} p u-e=\sum_{k=0}^{l} \sum_{i=1}^{r(k)} u^{*} q_{k, i} u .
$$

We have

$$
\left(u^{*} q_{k, i} u\right)\left(g_{k, i}^{(2)} \circ h\right)=u^{*} q_{k, i} g_{k, i}^{(2)} u=u^{*} q_{k, i} u,
$$

and because $\operatorname{supp}\left(g_{k, i}^{(2)} \circ h\right) \subset Y$ and $f$ vanishes on $Y$, we get $\left(g_{k, i}^{(2)} \circ h\right) f=0$. Therefore

$$
p u f-u e f=u\left(u^{*} p u-e\right) f=u \sum_{k=0}^{l} \sum_{i=1}^{r(k)} u^{*} q_{k, i} u\left(g_{k, i}^{(2)} \circ h\right) f=0 .
$$

Now

$\|p u f-u b\|=\|e f-b\| \leq\|e\| \cdot\|p f-b\| \leq \frac{1}{3} \varepsilon \quad$ and $\quad\|u f p-u b\|=\|f p-b\| \leq \frac{1}{3} \varepsilon$,

SO

$$
\|[p, u f]\| \leq \frac{2}{3} \varepsilon<\varepsilon \quad \text { and } \quad\|p u f p-u b\| \leq\|p u f-u b\| \cdot\|p\| \leq \frac{1}{3} \varepsilon<\varepsilon .
$$

This gives (15) and (16) for $a \in u \mathcal{F}_{0}$. The only other element of $\mathcal{F}$ is 1 , for which (15) and (16) are obvious. This completes the verification of (15) and (16).

Finally, we prove (17). Let $\tau$ be a tracial state on $A_{\{y\}}$. The restriction map $T(A) \rightarrow T\left(A_{\{y\}}\right)$ is bijective by Proposition 2.6 Therefore $\tau$ is the restriction to 
$A_{\{y\}}$ of the tracial state on $A$ obtained from some $h$-invariant Borel probability measure $\mu$. We have $p \geq \sum_{(k, i, j) \in Q} g_{k, i}^{(0)} \circ h^{-(j-1)}$. Therefore

$$
\begin{aligned}
\tau(p) & \geq \sum_{(k, i, j) \in Q} \int_{X} g_{k, i}^{(0)} \circ h^{-(j-1)} d \mu \geq \sum_{(k, i, j) \in Q} \mu\left(h^{j}\left(W_{k, i}\right)\right) \\
& =\mu((X \backslash \bar{V}) \cap U)=\mu(X \backslash \bar{V}) \geq 1-\mu\left(V_{0}\right)>1-\varepsilon .
\end{aligned}
$$

This proves (17), and completes the proof of the theorem.

\section{The tracial Rank of the of the transformation group $\mathrm{C}^{*}$-Algebra}

In this section, we prove the main result, Theorem 4.6 below.

The following lemma is a version of a result of L. G. Brown.

Lemma 4.1. Let $A$ be a $C^{*}$-algebra with real rank zero, and let $a_{1}, a_{2}, a_{3} \in A$ satisfy

$$
0 \leq a_{1} \leq a_{2} \leq a_{3} \leq 1, \quad a_{3} a_{2}=a_{2}, \quad \text { and } \quad a_{2} a_{1}=a_{1} .
$$

Then there exists a projection $e \in A$ such that

$$
e a_{1}=a_{1} e=a_{1} \quad \text { and } \quad a_{3} e=e a_{3}=e .
$$

Proof. Since the unitization of $A$ again has real rank zero, and since the equation $a_{3} e=e$ will imply $e \in A$, we may assume $A$ is unital. In $A^{* *}$ let $p$ and $q$ be the closed projections

$$
p=\chi_{[2 / 3, \infty]}\left(a_{2}\right) \text { and } q=\chi_{[0,1 / 3]}\left(a_{2}\right) .
$$

Then one checks that

$$
a_{3}(1-q)=1-q \text { and } \quad p a_{1}=a_{1} .
$$

Use Theorem 1 of [4] to find a projection $e \in A$ such that $p \leq e \leq 1-q$. This projection clearly satisfies the conclusion.

Lemma 4.2. Let $X$ be an infinite compact metric space, and let $h: X \rightarrow X$ be a minimal homeomorphism. Let $A=C^{*}(\mathbb{Z}, X, h)$, and adopt Notation 2.1. Let $y \in X$, and suppose that $A_{\{y\}}$ has real rank zero and stable rank one. Then for any $\varepsilon>0$ and any finite subset $\mathcal{F} \subset A$, there is a projection $p \in A_{\{y\}}$ such that:

(1) $\|p a-a p\|<\varepsilon$ for all $a \in \mathcal{F}$.

(2) $p a p \in p A_{\{y\}} p$ for all $a \in \mathcal{F}$.

(3) $\tau(1-p)<\varepsilon$ for all $\tau \in T(A)$.

Proof. We may assume that $\mathcal{F}=\mathcal{G} \cup\{u\}$ for some finite subset $\mathcal{G} \subset C(X)$.

Choose $N_{0} \in \mathbb{N}$ so large that $4 \pi / N_{0}<\varepsilon$. Choose $\delta_{0}>0$ with $\delta_{0}<\frac{1}{2} \varepsilon$ and so small that $d\left(x_{1}, x_{2}\right)<4 \delta_{0}$ implies $\left|f\left(x_{1}\right)-f\left(x_{2}\right)\right|<\frac{1}{4} \varepsilon$ for all $f \in \mathcal{G}$. Choose $\delta>0$ with $\delta \leq \delta_{0}$ and such that whenever $d\left(x_{1}, x_{2}\right)<\delta$ and $0 \leq n \leq N_{0}$, then $d\left(h^{-n}\left(x_{1}\right), h^{-n}\left(x_{2}\right)\right)<\delta_{0}$.

Since $h$ is minimal, there is $N>N_{0}+1$ such that $d\left(h^{N}(y), y\right)<\delta$. Choose $R \in \mathbb{N}$ so large that $R>\left(N+N_{0}+1\right) / \min (1, \varepsilon)$. Since $h$ is free, there is an open neighborhood $U$ of $y$ in $X$ such that

$$
h^{-N_{0}}(U), h^{-N_{0}+1}(U), \ldots, U, h(U), \ldots, h^{R}(U)
$$

are disjoint and all have diameter less than $\delta$. In particular, this is true with $N$ in place of $R$, and furthermore $\mu(U)<\varepsilon /\left(N+N_{0}+1\right)$ for every $h$-invariant Borel probability measure $\mu$. 
Choose continuous functions $g_{0}, g_{1}, g_{2}, f_{0}: X \rightarrow[0,1]$ such that

$$
g_{0}(y)=1, \quad g_{1} g_{0}=g_{0}, \quad g_{2} g_{1}=g_{1}, \quad f_{0} g_{2}=g_{2}, \quad \text { and } \quad \operatorname{supp}\left(f_{0}\right) \subset U .
$$

By Lemma 4.1 there exists a projection $q_{0} \in A_{\{y\}}$ such that

$$
g_{1} q_{0}=q_{0} g_{1}=g_{1} \quad \text { and } \quad f_{0} q_{0}=q_{0} f_{0}=q_{0} .
$$

For $-N_{0} \leq n \leq N$ set

$$
q_{n}=u^{n} q_{0} u^{-n}, \quad f_{n}=u^{n} f_{0} u^{-n}=f_{0} \circ h^{-n}, \quad \text { and } \quad U_{n}=h^{n}(U) .
$$

We have $\operatorname{supp}\left(f_{n}\right) \subset U_{n}$ and $f_{n} q_{n}=q_{n} f_{n}=q_{n}$, so the $q_{n}$ are mutually orthogonal projections in $A$.

We claim that $q_{n} \in A_{\{y\}}$ for $-N_{0} \leq n \leq N$. We first consider $q_{-n}$ for $1 \leq n \leq N_{0}$. For these $n$, we have $u f_{-n} \in A_{\{y\}}$, so

$$
a_{n}=f_{0}^{n} u^{n}=\left(u f_{-1}\right)\left(u f_{-2}\right) \cdots\left(u f_{-n}\right) \in A_{\{y\}} .
$$

Then $q_{-n}=u^{-n} q_{0} u^{n}=a_{n}^{*} q_{0} a_{n} \in A_{\{y\}}$, as desired.

Next, we show $q_{1} \in A_{\{y\}}$. From $q_{0} g_{0}=q_{0} g_{1} g_{0}=g_{1} g_{0}$ we get $\left(q_{0}-g_{1}\right)\left(f_{0}-g_{0}\right)=$ $q_{0}-g_{1}$. Also, $u\left(f_{0}-g_{0}\right) \in A_{\{y\}}$ because $\left(f_{0}-g_{0}\right)(y)=0$. Moreover, $u g_{1} u^{*}=$ $g_{1} \circ h^{-1} \in A_{\{y\}}$. So

$q_{1}=u q_{0} u^{*}=u\left(q_{0}-g_{1}\right) u^{*}+u g_{1} u^{*}=\left[u\left(f_{0}-g_{0}\right)\right]\left(q_{0}-g_{1}\right)\left[u\left(f_{0}-g_{0}\right)\right]^{*}+u g_{1} u^{*} \in A_{\{y\}}$.

For $2 \leq n \leq N$ we now have

$$
q_{n}=\left[\left(u f_{n}\right)\left(u f_{n-1}\right) \cdots\left(u f_{1}\right)\right] q_{1}\left[\left(u f_{n}\right)\left(u f_{n-1}\right) \cdots\left(u f_{1}\right)\right]^{*},
$$

which is in $A_{\{y\}}$ because $f_{1}, f_{2}, \ldots, f_{N}$ all vanish on $U$ and $y \in U$. This proves the claim.

We now have a sequence of projections, in principle going to infinity in both directions:

$$
\ldots, q_{-N_{0}}, \ldots, q_{-1}, q_{0}, q_{1}, \ldots, q_{N-N_{0}}, \ldots, q_{N-1}, q_{N}, \ldots
$$

The ones shown are orthogonal, and conjugation by $u$ is the shift. The projections $q_{0}$ and $q_{N}$ live over open sets which are disjoint but close to each other, and similarly for the pairs $q_{-1}$ and $q_{N-1}$ down to $q_{-N_{0}}$ and $q_{N-N_{0}}$. We are now going to use Berg's technique [1] to splice this sequence along the pairs of indices $\left(-N_{0}, N-\right.$ $N_{0}$ ) through $(0, N)$, obtaining a loop of length $N$ on which conjugation by $u$ is approximately the cyclic shift.

We claim that there is a partial isometry $w \in A_{\{y\}}$ such that $w^{*} w=q_{0}$ and $w w^{*}=q_{N}$. Certainly $\left[q_{0}\right]=\left[q_{N}\right]$ in $K_{0}(A)$. The map $K_{0}\left(A_{\{y\}}\right) \rightarrow K_{0}(A)$ is a group isomorphism by Theorem 4.1(3) of [35], so $\left[q_{0}\right]=\left[q_{N}\right]$ in $K_{0}\left(A_{\{y\}}\right)$. Since $A_{\{y\}}$ has stable rank one, Proposition 6.5.1 of 2$]$ implies that projections in matrix algebras over $A_{\{y\}}$ satisfy cancellation. The claim follows.

For $t \in \mathbb{R}$ define $v(t)=\cos (\pi t / 2)\left(q_{0}+q_{N}\right)+\sin (\pi t / 2)\left(w-w^{*}\right)$. Then $v(t)$ is a unitary in the corner $\left(q_{0}+q_{N}\right) A_{\{y\}}\left(q_{0}+q_{N}\right)$ whose matrix with respect to the obvious block decomposition is

$$
v(t)=\left(\begin{array}{cc}
\cos (\pi t / 2) & -\sin (\pi t / 2) \\
\sin (\pi t / 2) & \cos (\pi t / 2)
\end{array}\right) .
$$

For $0 \leq k \leq N_{0}$ define $w_{k}=u^{-k} v\left(k / N_{0}\right) u^{k}$. With $a_{k}$ as in (11) and with

$$
b_{k}=f_{N}^{k} u^{k}=\left(u f_{N-1}\right)\left(u f_{N-2}\right) \cdots\left(u f_{N-k}\right) \in A_{\{y\}}
$$


(because $N_{0}<N$ ), and using $U_{-k} \cap U_{N-k}=U_{0} \cap U_{N}=\varnothing$, we can write

$$
w_{k}=\left(a_{k}+b_{k}\right)^{*} v\left(k / N_{0}\right)\left(a_{k}+b_{k}\right) \in A_{\{y\}} .
$$

Therefore $w_{k}$ is a unitary in the corner $\left(q_{-k}+q_{N-k}\right) A_{\{y\}}\left(q_{-k}+q_{N-k}\right)$. Moreover, adding estimates on the differences of the matrix entries at the second step,

$$
\left\|u w_{k+1} u^{*}-w_{k}\right\|=\left\|v\left(k / N_{0}\right)-v\left((k-1) / N_{0}\right)\right\| \leq 2 \pi / N_{0}<\frac{1}{2} \varepsilon .
$$

Now define $e_{n}=q_{n}$ for $0 \leq n \leq N-N_{0}$, and for $N-N_{0} \leq n \leq N$ write $k=N-n$ and set $e_{n}=w_{k} q_{-k} w_{k}^{*}$. The two definitions for $n=N-N_{0}$ agree because $w_{N_{0}} q_{-N_{0}} w_{N_{0}}^{*}=q_{N-N_{0}}$, and moreover $e_{N}=e_{0}$. Therefore $u e_{n-1} u^{*}=e_{n}$ for $1 \leq n \leq N-N_{0}$, and also $u e_{N} u^{*}=e_{1}$, while for $N-N_{0}<n \leq N$ we have

$$
\left\|u e_{n-1} u^{*}-e_{n}\right\| \leq 2\left\|u w_{N-n+1} u^{*}-w_{N-n}\right\|<\varepsilon .
$$

Also, clearly $e_{n} \in A_{\{y\}}$ for all $n$.

Set $e=\sum_{n=1}^{N} e_{n}$ and $p=1-e$. We verify that $p$ satisfies (1) through (3).

First,

$$
p-u p u^{*}=u e u^{*}-e=\sum_{n=N_{0}+1}^{N}\left(u e_{n-1} u^{*}-e_{n}\right) .
$$

The terms in the sum are orthogonal and have norm less than $\varepsilon$, so $\left\|u p u^{*}-p\right\|<\varepsilon$. Furthermore, since

$$
1-g_{1} \in C_{0}(X \backslash\{y\}), \quad q_{0} g_{1}=g_{1}, \quad \text { and } \quad p \leq 1-q_{0},
$$

we get

$$
p u p=p u\left(1-g_{1}\right)\left(1-q_{0}\right) p \in A_{\{y\}} .
$$

This is (1) and (2) for the element $u \in \mathcal{F}$.

Next, let $g \in \mathcal{G}$. The sets $U_{0}, U_{1}, \ldots, U_{N}$ all have diameter less than $\delta$. We have $d\left(h^{N}(y), y\right)<\delta$, so the choice of $\delta$ implies that $d\left(h^{n}(y), h^{n-N}(y)\right)<\delta_{0}$ for $N-N_{0} \leq n \leq N$. Also, $U_{n-N}=h^{n-N}\left(U_{0}\right)$ has diameter less than $\delta$. Therefore $U_{n-N} \cup U_{n}$ has diameter less than $2 \delta+\delta_{0} \leq 3 \delta_{0}$. Since $g$ varies by at most $\frac{1}{4} \varepsilon$ on any set with diameter less than $4 \delta_{0}$, and since the sets

$$
U_{1}, U_{2}, \ldots, U_{N-N_{0}-1}, U_{N-N_{0}} \cup U_{-N_{0}}, U_{N-N_{0}+1} \cup U_{-N_{0}+1}, \ldots, U_{N} \cup U_{0}
$$

are disjoint, there is $\widetilde{g} \in C(X)$ which is constant on each of these sets and satisfies $\|g-\widetilde{g}\|<\frac{1}{2} \varepsilon$. Let the values of $\widetilde{g}$ on these sets be $\lambda_{1}$ on $U_{1}$ through $\lambda_{N}$ on $U_{N} \cup U_{0}$.

For $0 \leq n \leq N-N_{0}$ we have $f_{n} e_{n}=f_{n} q_{n}=q_{n}=e_{n}$, whence $\operatorname{supp}\left(f_{n}\right) \subset U_{n}$ implies

$$
\widetilde{g} e_{n}=\widetilde{g} f_{n} e_{n}=\lambda_{n} f_{n} e_{n}=\lambda_{n} e_{n}
$$

and similarly $e_{n} \widetilde{g}=\lambda_{n} e_{n}$. For $N-N_{0}<n \leq N$ we use $e_{n} \in\left(q_{n-N}+q_{n}\right) A_{\{y\}}\left(q_{n-N}+\right.$ $\left.q_{n}\right)$ to get, in the same way as above,

$$
\left(f_{n-N}+f_{n}\right) e_{n}=e_{n}\left(f_{n-N}+f_{n}\right)=e_{n},
$$

so from $\operatorname{supp}\left(f_{n-N}+f_{n}\right) \subset U_{n-N}+U_{n}$ we get $\widetilde{g} e_{n}=\lambda_{n} e_{n}=e_{n} \widetilde{g}$. Since $\|g-\widetilde{g}\|<\frac{1}{2} \varepsilon$, it follows that

$$
\|p g-g p\|=\|g e-e g\|<\varepsilon .
$$

This is (1) for $g$. That $p g p \in A_{\{y\}}$ follows from the fact that $g$ and $p$ are in this subalgebra. So we also have (2) for $g$. 
It remains only to verify (3). Let $\tau \in T(A)$, and let $\mu$ be the corresponding $h$-invariant probability measure on $X$. We have

$$
1-p=e \leq \sum_{n=-N_{0}}^{N} q_{n} \leq \sum_{n=-N_{0}}^{N} f_{n}
$$

so

$$
\tau(1-p) \leq \sum_{n=-N_{0}}^{N} \mu\left(U_{n}\right)=\sum_{n=-N_{0}}^{N} \mu\left(h^{n}(U)\right)=\left(N+N_{0}+1\right) \mu(U)<\varepsilon .
$$

This completes the proof.

The next two lemmas assert that for a simple $\mathrm{C}^{*}$-algebra, in Definition 1.1 of tracial rank zero, and in the definition of the local approximation property of Popa 39, the finite dimensional subalgebras can be replaced by subalgebras with tracial rank zero. We state these lemmas separately so as to have them available for use elsewhere. We do not require the subalgebra $B$ in the first to be simple, because it does not simplify the proof. However, for the application in this paper, $B$ will in fact be simple, so that the reader need not worry about the extra complexity of tracial rank for nonsimple algebras.

The first lemma is certainly known. The second is a special case of Theorem 4.6 of [14, where neither $A$ nor $B$ is required to be simple, but the proof here is much easier. This argument can also be found in the last part of the proof of Theorem 2.9 of $[27$.

Lemma 4.3. Let $A$ be a simple unital $\mathrm{C}^{*}$-algebra. Suppose that for every $\varepsilon>0$ and every finite subset $\mathcal{F} \subset A$, there exists a unital $\mathrm{C}^{*}$-subalgebra $B \subset A$ which has tracial rank zero and a projection $p \in B$ such that

$$
\|p a-a p\|<\varepsilon \quad \text { and } \operatorname{dist}(p a p, p B p)<\varepsilon
$$

for all $a \in \mathcal{F}$. Then $A$ has the local approximation property of Popa [39], that is, for every $\varepsilon>0$ and every finite subset $\mathcal{F} \subset A$, there exists a nonzero projection $q \in A$ and a finite dimensional unital $\mathrm{C}^{*}$-subalgebra $D \subset q A q$ such that

$$
\|q a-a q\|<\varepsilon \quad \text { and } \operatorname{dist}(q a q, D)<\varepsilon
$$

for all $a \in \mathcal{F}$.

Proof. Let $\varepsilon>0$ and let $\mathcal{F} \subset A$ be a finite subset. Choose $B$ and $p$ as in the hypotheses, with $\frac{1}{5} \varepsilon$ in place of $\varepsilon$. In particular, there exists a finite subset $\mathcal{G} \subset p B p$ such that $\operatorname{dist}($ pap, $\mathcal{G})<\frac{1}{5} \varepsilon$ for all $a \in \mathcal{F}$.

Since $B$ has tracial rank zero, Theorem 5.3 of [21 implies that $p B p$ also has tracial rank zero. So there is a nonzero projection $q \in p B p$ and a finite dimensional unital $\mathrm{C}^{*}$-subalgebra $D \subset q B q$ such that

$$
\|q b-b q\|<\frac{1}{5} \varepsilon \quad \text { and } \operatorname{dist}(q b q, D)<\frac{1}{5} \varepsilon
$$

all $b \in \mathcal{G}$. We claim that

$$
\|q a-a q\|<\varepsilon \quad \text { and } \operatorname{dist}(q a q, D)<\varepsilon
$$


for all $a \in \mathcal{F}$. The second part is easy. For the first, let $a \in \mathcal{F}$ and choose $b \in \mathcal{G}$ such that $\|q a q-b\|<\frac{1}{5} \varepsilon$. Then

$$
\begin{aligned}
\|q a-a q\| & =\|q p a-a p q\| \leq\|q p a p-p a p q\|+2\|p a-a p\| \\
& \leq\|q b-b q\|+2\|b-p a p\|+2\|p a-a p\|<\varepsilon .
\end{aligned}
$$

This completes the proof.

Lemma 4.4. Let $A$ be a simple unital $\mathrm{C}^{*}$-algebra. Suppose that for every finite subset $\mathcal{F} \subset A$, every $\varepsilon>0$, and every nonzero positive element $c \in A$, there exists a projection $p \in A$ and a simple unital subalgebra $B \subset p A p$ with tracial rank zero such that:

(1) $\|[a, p]\|<\varepsilon$ for all $a \in \mathcal{F}$.

(2) $\operatorname{dist}($ pap,$B)<\varepsilon$ for all $a \in \mathcal{F}$.

(3) $p$ is Murray-von Neumann equivalent to a projection in $\overline{c A c}$.

Then $A$ has tracial rank zero.

Proof. Even without (3), the algebra $A$ has Property (SP) by Lemma 2.12 of 20 . If $A$ is finite dimensional, the result is trivial; otherwise, there are nonzero orthogonal projections $e, f \in \overline{c A c}$.

The proof is now essentially the same as that of Lemma 4.3 The projection $p$ in that proof can be required to satisfy $1-p \precsim e$. Use Lemma 3.1 of [20] to find a nonzero projection $f_{0} \leq p$ with $f_{0} \precsim f$, and require the projection $q \in p B p$ to satisfy $p-q \precsim f_{0}$ in $p B p$.

Theorem 4.5. Let $X$ be an infinite compact metric space, and let $h: X \rightarrow X$ be a minimal homeomorphism. Let $A=C^{*}(\mathbb{Z}, X, h)$, and adopt Notation 2.1 Suppose that there is $y \in X$ such that $A_{\{y\}}$ has tracial rank zero. Then $A$ has tracial rank zero.

Proof. We verify the condition of Lemma 4.4 Thus, let $\mathcal{F} \subset A$ be a finite subset, let $\varepsilon>0$, and let $c \in A$ be a nonzero positive element. Beyond Lemma 4.2 the main step of the proof is to find a nonzero projection in $A_{\{y\}}$ which is Murray-von Neumann equivalent to a projection in $\overline{c A c}$.

The algebra $A$ is simple, so Lemma 4.2. Lemma 4.3] and Lemma 2.12 of 20] imply that $A$ has property (SP). Therefore there is a nonzero projection $e \in \overline{c A c}$. Set

$$
\delta_{0}=\frac{1}{18} \inf _{\tau \in T(A)} \tau(e) \leq \frac{1}{18} .
$$

By Lemma 4.2 there is a projection $q \in A_{\{y\}}$ and an element $b_{0} \in q A_{\{y\}} q$ such that

$$
\|q e-e q\|<\delta_{0}, \quad\left\|q e q-b_{0}\right\|<\delta_{0}, \quad \text { and } \sup _{\tau \in T(A)} \tau(1-q) \leq \delta_{0} .
$$

Replacing $b_{0}$ by $\frac{1}{2}\left(b_{0}+b_{0}^{*}\right)$, we may assume that $b_{0}$ is selfadjoint. We have $-\delta_{0} \leq$ $b_{0} \leq 1+\delta_{0}$, so applying continuous functional calculus we may find $b \in q A_{\{y\}} q$ such that $0 \leq b \leq 1$ and $\|q e q-b\|<2 \delta_{0}$. Using $\|q e-e q\|<\delta_{0}$ on the last term in the second expression, we get

$$
\left\|b^{2}-b\right\| \leq 3\|b-q e q\|+\left\|(q e q)^{2}-q e q\right\|<3 \cdot 2 \delta_{0}+\delta_{0}=7 \delta_{0}<\frac{1}{4} .
$$


Therefore there is a projection $e_{1} \in q A_{\{y\}} q$ such that $\left\|e_{1}-b\right\|<14 \delta_{0}$, giving $\| e_{1}-$ $q e q \|<16 \delta_{0}$. Similarly (actually, one gets a better estimate) there is a projection $e_{2} \in(1-q) A(1-q)$ such that $\left\|e_{2}-(1-q) e(1-q)\right\|<16 \delta_{0}$. Therefore

$$
\left\|e_{1}+e_{2}-[q e q+(1-q) e(1-q)]\right\|<16 \delta_{0}
$$

and, using $\|q e-e q\|<\delta_{0}$ again, we have $\left\|e_{1}+e_{2}-e\right\|<18 \delta_{0} \leq 1$. It follows that $e_{1} \precsim e$. Also, for $\tau \in T(A)$, we have

$\tau\left(e_{1}\right)>\tau(q e q)-16 \delta_{0}=\tau(e)-\tau((1-q) e(1-q))-16 \delta_{0} \geq \tau(e)-\tau(1-q)-16 \delta_{0}>0$, so $e_{1} \neq 0$.

Now set $\varepsilon_{0}=\inf \left(\left\{\tau\left(e_{2}\right): \tau \in T(A)\right\}\right)$. By Lemma 4.2 there is a projection $p \in A_{\{y\}}$ such that

(1) $\|p a-a p\|<\varepsilon$ for all $a \in \mathcal{F}$.

(2) pap $\in p A_{\{y\}} p$ for all $a \in \mathcal{F}$.

(3) $\tau(1-p)<\varepsilon_{0}$ for all $\tau \in T(A)$.

Using Proposition 2.6. it follows from (3) that $\tau(1-p)<\varepsilon_{0}$ for all $\tau \in T\left(A_{\{y\}}\right)$. Since $A_{\{y\}}$ has tracial rank zero, and since tracial rank zero implies that the order on projections is determined by traces (Theorems 6.8 and 6.13 of [21), it follows that $1-p \precsim e_{1} \precsim e$. Since $p A_{\{y\}} p$ also has tracial rank zero (Theorem 5.3 of [21]), we have verified the hypotheses of Lemma 4.4 Thus $A$ has tracial rank zero.

Theorem 4.6. Let $X$ be an infinite compact metric space with finite covering dimension, and let $h: X \rightarrow X$ be a minimal homeomorphism. Suppose that $\rho\left(K_{0}(A)\right)$ is dense in $\operatorname{Aff}(T(A))$. Then $C^{*}(\mathbb{Z}, X, h)$ is a simple unital $\mathrm{C}^{*}$-algebra with tracial rank zero which satisfies the Universal Coefficient Theorem.

Proof. It is well known that $C^{*}(\mathbb{Z}, X, h)$ is simple and unital.

By Theorem 4.5 for tracial rank zero it suffices to show that $A_{\{y\}}$ has tracial rank zero for some $y \in X$. The existence of $y$ with this property follows from Theorem 3.7

It follows from Theorem 1.17 of 44, and the description of the class $\mathcal{N}$ preceding its statement, that the crossed product of any commutative $\mathrm{C}^{*}$-algebra by any action of $\mathbb{Z}$ satisfies the Universal Coefficient Theorem.

Corollary 4.7. Let $X$ be an infinite compact metric space with finite covering dimension, and let $h: X \rightarrow X$ be a minimal homeomorphism. Suppose that $\rho\left(K_{0}(A)\right)$ is dense in $\operatorname{Aff}(T(A))$. Then $C^{*}(\mathbb{Z}, X, h)$ is a simple AH algebra with no dimension growth and with real rank zero.

Proof. This follows from Theorem 5.2 of [22] and Theorem 4.6] together with the existence of a simple AH algebra with the required Elliott invariant. See Lemma 7.5 of 37. for how to put everything together.

The converses of Theorem 4.6 and Corollary 4.7 are of course easy. (See Proposition $1.10(\mathrm{a})$ of [36.)

Corollary 4.8. For $j=1,2$ let $X_{j}$ be an infinite compact metric space with finite covering dimension, and let $h_{j}: X \rightarrow X$ be a minimal homeomorphism. Let $A_{j}=C^{*}\left(\mathbb{Z}, X, h_{j}\right)$. Suppose that $\rho_{A_{j}}\left(K_{0}\left(A_{j}\right)\right)$ is dense in $\left.\operatorname{Aff}\left(T\left(A_{j}\right)\right)\right)$ for $j=1,2$. Then $A_{1} \cong A_{2}$ if and only if

$$
\left.\left(K_{0}\left(A_{1}\right), K_{0}\left(A_{1}\right)_{+},\left[1_{A_{1}}\right], K_{1}\left(A_{1}\right)\right) \cong K_{0}\left(A_{2}\right), K_{0}\left(A_{2}\right)_{+},\left[1_{A_{2}}\right], K_{1}\left(A_{2}\right)\right) .
$$


Proof. This is immediate from Theorem 5.2 of [22] and Theorem 4.6

\section{EXAMPLES}

In this section, we describe one general type of example to which our result applies, and then prove the isomorphism of four fairly specific pairs of transformation group $\mathrm{C}^{*}$-algebras.

For our general example, we recall briefly the theory of rotation numbers for automorphisms developed in 9 . Let $X$ be a connected compact metric space, let $h: X \rightarrow X$ be a homeomorphism, and let $\mu$ be an $h$-invariant Borel measure on $X$. (Exel works more generally with a unital $\mathrm{C}^{*}$-algebra $A$, an automorphism $\alpha: A \rightarrow$ $A$, and an $\alpha$-invariant tracial state $\tau$ on $A$ such that $\tau_{*}\left(K_{0}(A)\right) \subset \mathbb{Z}$.) Then the rotation number $\rho_{h}^{\mu}$ associated with $h$ and $\mu$ (Definition IV.1 of [9]) is a homomorphism with domain the kernel of the homomorphism id $-\left(h^{-1}\right)^{*}: K^{1}(X) \rightarrow K^{1}(X)$ and codomain $\mathbb{R} / \mathbb{Z}$. It is defined as follows. As usual, let $\alpha: C(X) \rightarrow C(X)$ be the automorphism $\alpha(f)=f \circ h^{-1}$. Let $u \in U\left(M_{n}(C(X))\right.$ satisfy $\left(\right.$ id $\left.-\left(h^{-1}\right)^{*}\right)([u])=0$. Let $v=\alpha\left(u^{*}\right) u$. Then $[v]=0$ in $K_{1}(C(X))$. Increasing the matrix size and replacing $u$ by $\operatorname{diag}(u, 1)$, we may assume that $v \in U_{0}\left(M_{n}(C(X))\right.$. Then there exist $a_{1}, a_{2}, \ldots, a_{m} \in M_{n}(C(X))_{\text {sa }}$ such that $\prod_{k=1}^{m} e^{i a_{k}}=v$. Now

$$
\rho_{h}^{\mu}([u])=\mathbb{Z}+\frac{1}{2 \pi} \int_{X} \sum_{k=1}^{m} \operatorname{Tr}\left(a_{k}(x)\right) d \mu(x) .
$$

See Section IV of 9 for the details, including the proof that $\rho_{h}^{\mu}$ is well defined; see Definition II.2 of [9] for the determinant used there.

Definition 5.1. Let $X$ be a connected compact metric space, and let $h: X \rightarrow X$ be a homeomorphism. We say that $h$ has an irrational rotation number if there exists $\eta$ in the kernel of id $-\left(h^{-1}\right)^{*}: K^{1}(X) \rightarrow K^{1}(X)$ and an $h$-invariant Borel probability measure $\mu$ on $X$ such that, with $\rho_{h}^{\mu}$ as above, one has $\rho_{h}^{\mu}(\eta) \notin \mathbb{Q} / \mathbb{Z}$.

By Theorem VI.11 of [9], one only needs to consider $\eta$ of the form $[u]$ with $u \in C(X)$.

If $X=S^{1}$, if $h(\zeta)=\exp (2 \pi i \theta) \zeta$ for $\zeta \in S^{1}$, and if $\mu$ is normalized Haar measure, then the unitary $u(\zeta)=\zeta$ has $\rho_{h}^{\mu}([u])=\theta+\mathbb{Z}$. See Example IV.5 of $[9]$.

In this section, we are particularly interested in the uniquely ergodic case.

Lemma 5.2. Let $X$ be a connected compact metric space, let $h: X \rightarrow X$ be a minimal homeomorphism, and let $A=C^{*}(\mathbb{Z}, X, h)$. Suppose that $h$ is uniquely ergodic and has an irrational rotation number. Then $\rho\left(K_{0}(A)\right)$ is dense in $\operatorname{Aff}(T(A))$.

Proof. We identify $\operatorname{Aff}(T(A))$ with $\mathbb{R}$. Clearly $\rho\left(K_{0}(A)\right)$ contains $\mathbb{Z}$. It follows from Theorem V.12 of $\left[9\right.$ that the image of $\rho\left(K_{0}(A)\right)$ in $\mathbb{R} / \mathbb{Z}$ contains $\theta+\mathbb{Z}$ for some $\theta \notin \mathbb{Q}$. So $\rho\left(K_{0}(A)\right)$ contains $\mathbb{Z}+\theta \mathbb{Z}$, which is dense in $\mathbb{R}$.

Corollary 5.3. Let $X$ be a finite dimensional connected compact metric space and let $h: X \rightarrow X$ be a minimal homeomorphism. Suppose that $(X, h)$ is uniquely ergodic and has an irrational rotation number. Then $C^{*}(\mathbb{Z}, X, h)$ is a simple unital $\mathrm{AH}$ algebra with no dimension growth and with real rank zero.

Proof. Combine Lemma 5.2 and Corollary 4.7 
Example 5.4. Corollary [5.3 allows us to recover the Elliott-Evans Theorem [7], stating that the irrational rotation algebra $A_{\theta}$ is an AT algebra with real rank zero. As noted after Definition 5.1 an irrational rotation has an irrational rotation number, and it is well known that every irrational rotation is uniquely ergodic. So $A_{\theta}$ is a simple unital AH algebra with no dimension growth and with real rank zero. Since its K-theory is torsion free (easily obtained from the Pimsner-Voiculescu exact sequence 38), it follows from known results on classification and the range of the Elliott invariant that $A_{\theta}$ is an AT algebra. (See Lemma 7.5 of [37 for how to put the results together to get this conclusion.)

Remark 5.5. Let $X$ be a connected compact metric space such that $K^{1}(X)$ is finitely generated. (For example, $X$ could be a finite CW complex.) Let $h: X \rightarrow X$ be a minimal homeomorphism. Suppose that $C^{*}(\mathbb{Z}, X, h)$ has tracial rank zero, or even just suppose that $C^{*}(\mathbb{Z}, X, h)$ has real rank zero. Then we claim that $h$ has an irrational rotation number.

To see this, let $A=C^{*}(\mathbb{Z}, X, h)$. Let $\mu$ be any $h$-invariant Borel probability measure on $X$, and let $\tau$ be the corresponding trace on $A$. The map $\rho: K_{0}(A) \rightarrow$ $\operatorname{Aff}(T(A))$ has dense range by Proposition 1.10(a) of [36. In particular, $\tau_{*}\left(K_{0}(A)\right)$ is dense in $\mathbb{R}$. Let $\iota: C(X) \rightarrow A$ be the standard inclusion, and let $G \subset K^{1}(X)$ be the kernel of the map id $-\left(h^{-1}\right)^{*}: K^{1}(X) \rightarrow K^{1}(X)$. From the Pimsner-Voiculescu exact sequence [38], we get an exact sequence as the top row of the following diagram. The square commutes by Theorem V.12 of $[9]$.

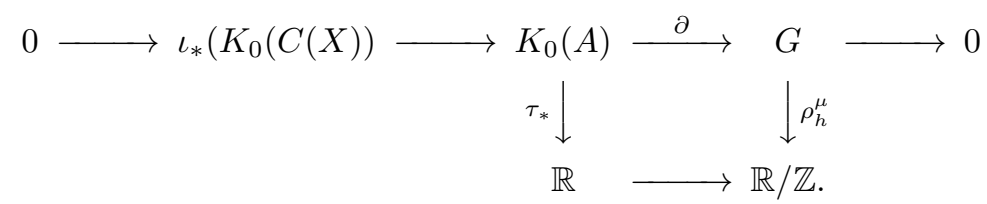

Since $X$ is connected, $\tau_{*}\left(\iota_{*}\left(K_{0}(C(X))\right)\right)=\mathbb{Z}$. Therefore $\rho_{h}^{\mu}$ has dense range. Since $G$ is finitely generated, this can only happen if its range is not contained in $\mathbb{Q} / \mathbb{Z}$.

We now turn specific examples of isomorphisms of transformation group $\mathrm{C}^{*}$ algebras. Except for Example [5.7 these were discussed in [33, although they predate that paper; the expected isomorphisms could not be proved there because of insufficient smoothness.

The first two we consider are covered by Corollary 5.3 but in fact the computations of the ordered K-theory have already been done.

Example 5.6. For each $\theta \in[0,1] \backslash \mathbb{Q}$, each continuous function $f: S^{1} \rightarrow \mathbb{R}$, and each $d \in \mathbb{Z} \backslash\{0\}$, we define $h=h_{\theta, f, d}: S^{1} \times S^{1} \rightarrow S^{1} \times S^{1}$ to be the inverse of the homeomorphism

$$
\left(\zeta_{1}, \zeta_{2}\right) \mapsto\left(e^{2 \pi i \theta} \zeta_{1}, e^{2 \pi i f\left(\zeta_{1}\right)} \zeta_{1}^{d} \zeta_{2}\right) .
$$

(One sees that the given map does in fact have a continuous inverse by writing down an explicit formula for it. This homeomorphism is called $\varphi_{f, \theta}$ in [17.) The homeomorphism $h$ is minimal by the remark after Theorem 2.1 in Section 2.3 of [10. Normalized Lebesgue measure on $S^{1} \times S^{1}$ is invariant, and when $f$ is Lipschitz this is the only invariant probability measure (Theorem 2.1 of [10]).

Let $B_{\theta, f, d}=C^{*}\left(\mathbb{Z}, S^{1} \times S^{1}, h\right)$ be the crossed product $\mathrm{C}^{*}$-algebra. The Elliott invariant of $B_{\theta, f, d}$ is computed in [17, and by a more direct method in Example 4.9 of [35]. It is described as follows. Let $A_{\theta}$ be the irrational rotation algebra. The map $S^{1} \times S^{1} \rightarrow S^{1}$ given by projection on the first coordinate intertwines $h$ and 
the irrational rotation $\zeta \mapsto e^{-2 \pi i \theta} \zeta$, and therefore gives a homomorphism $\varphi: A_{\theta} \rightarrow$ $B_{\theta, f, d}$. Let $p \in A_{\theta}$ be a projection for which the unique tracial state $\tau$ on $A_{\theta}$ satisfies $\tau(p)=\theta$, and let $\beta \in K_{0}\left(B_{\theta, f, d}\right)$ be the image of the Bott element in $K_{0}\left(C\left(S^{1} \times S^{1}\right)\right)$ under the inclusion. Then

$$
K_{0}\left(B_{\theta, f, d}\right) \cong \mathbb{Z}[1] \oplus \mathbb{Z} \beta \oplus \mathbb{Z}[\varphi(p)],
$$

every tracial state $\tau$ on $B_{\theta, f, d}$ satisfies

$$
\tau_{*}\left(m_{1}[1]+m_{2} \beta+m_{3}[\varphi(p)]\right)=m_{1}+m_{3} \theta,
$$

the positive part of $K_{0}$ is given by

$$
K_{0}\left(B_{\theta, f, d}\right)_{+}=\left\{m_{1}[1]+m_{2} \beta+m_{3}[\varphi(p)]: m_{1}+m_{3} \theta>0 \text { or } m_{1}=m_{2}=m_{3}=0\right\},
$$

and

$$
K_{1}\left(B_{\theta, f, d}\right) \cong \mathbb{Z}^{3} \oplus \mathbb{Z} / d \mathbb{Z}
$$

Although this is not explicitly stated in Example 4.9 of [35], one sees easily from the computations done there that the image of $K_{1}\left(C\left(S^{1} \times S^{1}\right)\right)$ in $K_{1}\left(B_{\theta, f, d}\right)$ is a direct summand, isomorphic to $\mathbb{Z} \oplus \mathbb{Z} / d \mathbb{Z}$, with $\mathbb{Z}$ generated by the image of the class of the unitary $u\left(\zeta_{1}, \zeta_{2}\right)=\zeta_{1}$ and $\mathbb{Z} / d Z$ generated by the image of the class of the unitary $u\left(\zeta_{1}, \zeta_{2}\right)=\zeta_{2}$.

Whenever $h$ is uniquely ergodic, Theorem 4.5 applies. In particular, whenever $f_{1}$ and $f_{2}$ are Lipschitz and $\left|d_{1}\right|=\left|d_{2}\right|$, it follows from Corollary 4.8 that $B_{\theta, f_{1}, d_{1}} \cong$ $B_{\theta, f_{2}, d_{2}}$.

When $d= \pm 1$, the K-theory is torsion free, so it follows as in Example 5.4 that $B_{\theta, f, d}$ is a simple AT algebra with real rank zero.

Example 5.7. Example 3.6 of [15] proves the existence of minimal homeomorphisms $h_{1}: X_{1} \rightarrow X_{1}$ and $h_{2}: X_{2} \rightarrow X_{2}$ of connected one dimensional compact metric spaces (which are suspension spaces of minimal homeomorphisms of the Cantor set and locally look like the product of an interval and the Cantor set) such that $C^{*}\left(\mathbb{Z}, X_{1}, h_{1}\right)$ and $C^{*}\left(\mathbb{Z}, X_{2}, h_{2}\right)$ have the same Elliott invariant but such that $h_{1}$ and $h_{2}$ are not flip conjugate. By Theorem 3.1 and Remark 3.4 of 3 , or by Proposition 5.5 of [29, $h_{1}$ and $h_{2}$ are not even topologically orbit equivalent. However, the Elliott invariants computed in [15] satisfy the dense range condition in Corollary 4.8 so this theorem implies that $C^{*}\left(\mathbb{Z}, X_{1}, h_{1}\right) \cong C^{*}\left(\mathbb{Z}, X_{2}, h_{2}\right)$.

The remaining examples involve disconnected spaces, so arguments using Exel's rotation numbers don't apply. The first is from Gjerde and Johansen [11, and is discussed in Example 1.6 of [33].

Example 5.8. It is shown in Theorem 4 of 11 that every minimal homeomorphism $h$ of the Cantor set $X$ has an almost one to one extension which is a minimal homeomorphism $k$ of a nonhomogeneous space $Y$ with covering $\operatorname{dimension} \operatorname{dim}(Y)=1$, such that $C^{*}(\mathbb{Z}, X, h)$ and $C^{*}(\mathbb{Z}, Y, k)$ have isomorphic Elliott invariants. It follows from Corollary 4.8 that, even though $h$ and $k$ are not even topologically orbit equivalent, we nevertheless have $C^{*}(\mathbb{Z}, Y, k) \cong C^{*}(\mathbb{Z}, X, h)$.

In particular, $C^{*}(\mathbb{Z}, Y, k)$ is a simple AT algebra with real rank zero.

The following example of Putnam appears as Example 1.7 of 33. We refer to 33 for a more detailed description and the computation of the Elliott invariants of the crossed products. 
Example 5.9. For any $\theta \in \mathbb{R} \backslash \mathbb{Q}$ let $r_{\theta}: S^{1} \rightarrow S^{1}$ be rotation by $2 \pi \theta$, and let $g_{\theta}$ be the minimal homeomorphism of a Cantor set $X_{\theta}$ obtained by disconnecting $S^{1}$ along a single orbit of $r_{\theta}$. (This orbit is the unique minimal set of a suitable Denjoy homeomorphism of $S^{1}$, as described in Example 1.7 of 33.) Now $\theta_{1}, \theta_{2} \in \mathbb{R} \backslash \mathbb{Q}$ be numbers such that $1, \theta_{1}, \theta_{2}$ are linearly independent over $\mathbb{Q}$. Define homeomorphisms

and

$$
h_{1}=r_{\theta_{1}} \times g_{\theta_{2}}: S^{1} \times X_{\theta_{2}} \rightarrow S^{1} \times X_{\theta_{2}}
$$

$$
h_{2}=r_{\theta_{2}} \times g_{\theta_{1}}: S^{1} \times X_{\theta_{1}} \rightarrow S^{1} \times X_{\theta_{1}} .
$$

Then, as shown in 33, the $\mathrm{C}^{*}$-algebras $C^{*}\left(\mathbb{Z}, S^{1} \times X_{\theta_{2}}, h_{1}\right)$ and $C^{*}\left(\mathbb{Z}, S^{1} \times X_{\theta_{1}}, h_{2}\right)$ have the same Elliott invariants, and they satisfy the dense range condition in Corollary 4.8 So this theorem implies that $C^{*}\left(\mathbb{Z}, S^{1} \times X_{\theta_{2}}, h_{1}\right) \cong C^{*}\left(\mathbb{Z}, S^{1} \times X_{\theta_{1}}, h_{2}\right)$, even though, as shown in Example 1.7 of [33, $h_{1}$ and $h_{2}$ are not topologically orbit equivalent.

Since the K-theory is torsion free (Proposition 1.12 of [33]), it follows as in Example 5.4 that the $\mathrm{C}^{*}$-algebras of such homeomorphisms are simple AT algebras with real rank zero.

\section{Approximate conjugacy}

Tomiyama has proved (see the corollary at the end of [45]) that two topologically transitive homeomorphisms $h_{1}$ and $h_{2}$ of compact metric spaces $X_{1}$ and $X_{2}$ are flip conjugate if and only if there exists an isomorphism $\varphi: C\left(\mathbb{Z}, X_{1}, h_{1}\right) \rightarrow C\left(\mathbb{Z}, X_{2}, h_{2}\right)$ which sends the canonical copy of $C\left(X_{1}\right)$ in the first algebra isomorphically to the canonical copy of $C\left(X_{2}\right)$ in the second algebra. In particular, this applies to minimal homeomorphisms. Motivated by this result, the first author introduced (Definition 3.7 of 24 ) the following definition.

Definition 6.1. Let $h_{1}: X_{1} \rightarrow X_{1}$ and $h_{2}: X_{2} \rightarrow X_{2}$ be minimal homeomorphisms of infinite compact metric spaces $X_{1}$ and $X_{2}$. Suppose that $A_{1}=C^{*}\left(\mathbb{Z}, X_{1}, h_{1}\right)$ and $A_{2}=C^{*}\left(\mathbb{Z}, X_{2}, h_{2}\right)$ have tracial rank zero. Let $\iota_{1}: C\left(X_{1}\right) \rightarrow A_{1}$ and $\iota_{2}: C\left(X_{2}\right) \rightarrow$ $A_{2}$ be the canonical embeddings. We say that $h_{1}$ and $h_{2}$ are $\mathrm{C}^{*}$-strongly approximately flip conjugate if there exists an isomorphism $\varphi: A_{1} \rightarrow A_{2}$, a homeomorphism $k: X_{1} \rightarrow X_{2}$, and a sequence of unitaries $u_{n} \in U\left(A_{2}\right)$ such that

$$
\lim _{n \rightarrow \infty}\left\|\operatorname{Ad}\left(u_{n}\right) \circ \iota_{1}(f)-\iota_{2}(f \circ k)\right\|=0
$$

for all $f \in C\left(X_{1}\right)$.

The next theorem is taken from [24]. Before stating it, we introduce some terminology. Recall Rørdam's $K L$-theory, as defined (for separable $\mathrm{C}^{*}$-algebras which satisfy the Universal Coefficient Theorem) after Lemma 5.3 in [43. Suppose that $A_{1}$ and $A_{2}$ have tracial rank zero and satisfy the Universal Coefficient Theorem, and that $\eta \in K L\left(A_{1}, A_{2}\right)$ gives a unit preserving order isomorphism $\Gamma(\eta)_{0}: K_{0}\left(A_{1}\right) \rightarrow K_{0}\left(A_{2}\right)$. The state space of $K_{0}\left(A_{j}\right)$ is exactly the space of quasitraces on $A_{j}$ (Theorem 6.9.1 of [2]), and it is easy to see from the definition (see 4.9(ix) of [23]) that if a $\mathrm{C}^{*}$-algebra $A$ has tracial rank zero, then all quasitraces on $A$ are in fact traces. So $\eta$ induces a unit preserving affine order isomorphism $L(\eta): \operatorname{Aff}\left(T\left(A_{1}\right)\right) \rightarrow \operatorname{Aff}\left(T\left(A_{2}\right)\right)$.

We also define $\rho_{A}: A_{\text {sa }} \rightarrow \operatorname{Aff}(T(A))$ by $\rho_{A}(a)(\tau)=\tau(a)$ for $\tau \in T(A)$; no confusion should arise with the map from $K_{0}(A)$ with the same name. 
Theorem 6.2. Let the notation and assumptions be as in Definition 6.1 Then $h_{1}$ and $h_{2}$ are $\mathrm{C}^{*}$-strongly approximately flip conjugate if and only if there exists a sequence of isomorphisms $\chi_{n}: C\left(X_{1}\right) \rightarrow C\left(X_{2}\right)$ and an element $\eta \in K L\left(A_{1}, A_{2}\right)$ such that:

(1) $\Gamma(\eta): K_{*}(A) \rightarrow K_{*}(B)$ is a unit preserving order isomorphism.

(2) $\eta \times\left[\iota_{1}\right]=\left[\iota_{2}\right] \times\left[\chi_{n}\right]$ in $K L\left(C\left(X_{1}\right), A_{2}\right)$ for all $n$.

(3) $\lim _{n \rightarrow \infty}\left\|\rho_{A_{2}} \circ \iota_{2} \circ \chi_{n}(f)-L(\eta) \circ \iota_{1}(f)\right\|=0$ for all $f \in C(X)_{\mathrm{sa}}$.

Corollary 6.3. Let $h_{1}: X_{1} \rightarrow X_{1}$ and $h_{2}: X_{2} \rightarrow X_{2}$ be minimal homeomorphisms of finite dimensional infinite compact metric spaces $X_{1}$ and $X_{2}$. Set $A_{j}=$ $C^{*}\left(\mathbb{Z}, X_{j}, h_{j}\right)$, and assume that $\rho_{A_{j}}\left(K_{0}\left(A_{j}\right)\right)$ is dense in $\operatorname{Aff}\left(T\left(A_{j}\right)\right)$ for $j=1,2$, and that $K^{*}\left(X_{j}\right)$ and $K_{*}\left(A_{j}\right)$ are torsion free for $j=1,2$. Suppose that there is a unit preserving order isomorphism

$$
\gamma:\left(K_{0}\left(A_{1}\right), K_{0}\left(A_{1}\right)_{+},\left[1_{A_{1}}\right], K_{1}\left(A_{1}\right)\right) \rightarrow\left(K_{0}\left(A_{2}\right), K_{0}\left(A_{2}\right)_{+},\left[1_{A_{2}}\right], K_{1}\left(A_{2}\right)\right)
$$

and an isomorphism $\chi: C\left(X_{1}\right) \rightarrow C\left(X_{2}\right)$ such that

$$
\gamma \circ\left(\iota_{1}\right)_{*}=\left(\iota_{2}\right)_{*} \circ \chi_{*} \text { and }\left.L(\gamma) \circ \rho_{A_{1}} \circ \iota_{1}\right|_{C\left(X_{1}\right)_{\mathrm{sa}}}=\left.\rho_{A_{2}} \circ \iota_{2} \circ \chi\right|_{C\left(X_{1}\right)_{\mathrm{sa}}} .
$$

Then $h_{1}$ and $h_{2}$ are $\mathrm{C}^{*}$-strongly approximately flip conjugate.

Proof. By Theorem 4.6] the algebras $A_{1}$ and $A_{2}$ have tracial rank zero. Since $K_{*}\left(A_{j}\right)$ is torsion free, the Universal Coefficient Theorem implies that $K L\left(A_{1}, A_{2}\right)=$ $\operatorname{Hom}\left(K_{*}\left(A_{1}\right), K_{*}\left(A_{2}\right)\right)$. (See the discussion before Lemma 5.2 and before and after Lemma 5.3 in 43.) Similarly, since $K_{*}\left(C\left(X_{1}\right)\right)$ is torsion free, $K L\left(C\left(X_{1}\right), A_{2}\right)=$ $\operatorname{Hom}\left(K_{*}\left(C\left(X_{1}\right), K_{*}\left(A_{2}\right)\right)\right.$. Now the corollary follows from Theorem 6.2

Example 6.4. Let $\theta \in[0,1] \backslash \mathbb{Q}$, let $f_{1}, f_{2}: S^{1} \rightarrow \mathbb{R}$ be continuous functions, and let $d_{1}, d_{2} \in \mathbb{Z} \backslash\{0\}$ satisfy $\left|d_{1}\right|=\left|d_{2}\right|$. Let $h_{1}=h_{\theta, f_{1}, d_{1}}$ and $h_{2}=h_{\theta, f_{2}, d_{2}}$ be uniquely ergodic Furstenberg transformations on $X=S^{1} \times S^{1}$ as in Example [5.6] and let $A_{1}=C^{*}\left(\mathbb{Z}, X, h_{1}\right)$ and $A_{2}=C^{*}\left(\mathbb{Z}, X, h_{2}\right)$. Let $\iota_{1}: C(X) \rightarrow A_{1}$ and $\iota_{2}: C(X) \rightarrow$ $A_{2}$ be the canonical embeddings. From the K-theory computation described in Example [5.6] one sees that there is an isomorphism $\gamma: K_{*}\left(A_{1}\right) \rightarrow K_{*}\left(A_{2}\right)$ which is an order isomorphism on $K_{0}$ and such that the following diagram commutes:

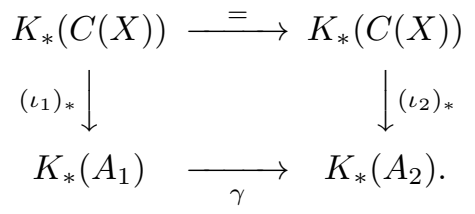

It follows from Theorem 5.2 of 22 that there is an isomorphism $\varphi: A_{1} \rightarrow A_{2}$ such that $\varphi_{*}=\gamma$.

Let $\tau_{1}$ and $\tau_{2}$ be the unique tracial states on $A_{1}$ and $A_{2}$. We claim that $\tau_{2} \circ \varphi \circ \iota_{1}=$ $\tau_{2} \circ \iota_{2}$. By uniqueness, we have $\tau_{2} \circ \varphi=\tau_{1}$. Let $\mu$ be normalized Lebesgue measure on $S^{1} \times S^{1}$, which is the unique ergodic measure for both $h_{2}$ and $h_{2}$. Then, for $f \in C(X)$, we have

$$
\tau_{2} \circ \varphi\left(\iota_{1}(f)\right)=\tau_{1}\left(\iota_{1}(f)\right)=\int_{S^{1} \times S^{1}} f d \mu=\tau_{2}\left(\iota_{2}(f)\right) .
$$

This proves the claim. It now follows from Corollary 6.3 that $h_{1}$ and $h_{2}$ are $\mathrm{C}^{*}$ strongly approximately flip conjugate. 
Suppose that $f_{1}=0$ and $d_{1}=d_{2}=1$. It has been shown in Example 2.1 of 33. that there are choices of $f_{2}$ for which $h_{1}$ and $h_{2}$ are not flip conjugate, and even not topologically orbit equivalent.

Remark 6.5. Let $X$ be the Cantor set and $h_{1}, h_{2}: X \rightarrow X$ be two minimal homeomorphisms. Then Corollary 6.3 implies that $h_{1}$ and $h_{2}$ are $\mathrm{C}^{*}$-strongly approximately flip conjugate if and only if there is a unit preserving order isomorphism between the K-groups $K^{0}\left(X, h_{1}\right)$ and $K^{0}\left(X, h_{2}\right)$ of Definition 1.11 of [12. In [25], it is shown this is also equivalent to the existence of sequences $\left(k_{n}\right)_{n \in \mathbb{N}}$ and $\left(l_{n}\right)_{n \in \mathbb{N}}$ of homeomorphisms from $X$ to $X$ such that:

(1) For all $f \in C(X)$, we have

$$
\lim _{n \rightarrow \infty}\left\|f \circ k_{n}^{-1} \circ h_{2} \circ k_{n}-f \circ h_{1}\right\|=0 \text { and } \lim _{n \rightarrow \infty}\left\|f \circ l_{n}^{-1} \circ h_{1} \circ l_{n}-f \circ h_{2}\right\|=0
$$

(2) For every projection $p \in C(X)$, for all sufficiently large $n$, the images of $k_{n}^{*}([p])$ and $k_{n+1}^{*}([p])$ in $K_{0}\left(C^{*}\left(\mathbb{Z}, X, h_{1}\right)\right.$ are equal; similarly, for every projection $q \in C(X)$, for all sufficiently large $n$, the images of $l_{n}^{*}([q])$ and $l_{n+1}^{*}([q])$ in $K_{0}\left(C^{*}\left(\mathbb{Z}, X, h_{2}\right)\right.$ are equal.

If such $\left(k_{n}\right)_{n \in \mathbb{N}}$ and $\left(l_{n}\right)_{n \in \mathbb{N}}$ exist, we say $\left(X, h_{1}\right)$ and $\left(X, h_{2}\right)$ are approximately $K$ conjugate. Approximate $K$-conjugacy is also equivalent to strong orbit equivalence as in [12]. (See [26].)

Approximate $K$-conjugacy can also be defined for more general spaces. See [25], 24], and [26]. In 26], approximate $K$-conjugacy has been studied for homeomorphisms of the product of the Cantor set and the circle.

We would like to ask the following question. Let $\left(X_{1}, h_{1}\right)$ and $\left(X_{2}, h_{2}\right)$ be two minimal systems, and let $A_{1}=C^{*}\left(\mathbb{Z}, X_{1}, h_{1}\right)$ and $A_{2}=C^{*}\left(\mathbb{Z}, X_{2}, h_{2}\right)$. Suppose that $\rho\left(K_{0}\left(A_{1}\right)\right)$ and $\rho\left(K_{0}\left(A_{2}\right)\right)$ are dense in $\operatorname{Aff}\left(T\left(A_{1}\right)\right)$ and $\operatorname{Aff}\left(T\left(A_{2}\right)\right)$, and that $h_{1}$ and $h_{2}$ are $\mathrm{C}^{*}$-strongly approximately flip conjugate. What additional hypotheses are required for $h_{1}$ and $h_{2}$ to be approximately $K$-conjugate?

\section{REFERENCES}

[1] I. D. Berg, On approximation of normal operators by weighted shifts, Michigan Math. J. 21(1974), 377-383.

[2] B. Blackadar, K-Theory for Operator Algebras, MSRI Publication Series 5, Springer-Verlag, New York, Heidelberg, Berlin, Tokyo, 1986.

[3] M. Boyle and J. Tomiyama, Bounded topological orbit equivalence and $C^{*}$-algebras, J. Math. Soc. Japan 50(1998), 317-329.

[4] L. G. Brown, Interpolation by projections in $C^{*}$-algebras of real rank zero, J. Operator Theory 26(1991), 383-387.

[5] A. Connes, An analogue of the Thom isomorphism for crossed products of a $C^{*}$-algebra by an action of $\mathbb{R}$, Advances in Math. 39(1981), 31-55.

[6] G. A. Elliott, The classification problem for amenable $C^{*}$-algebras, pages 922-932 in: Proceedings of the International Congress of Mathematicians, Zürich, 1994, S. D. Chatterji, ed., Birkhäuser, Basel, 1995.

[7] G. A. Elliott and D. E. Evans, The structure of the irrational rotation algebra, Ann. of Math. (2) 138(1993), 477-501.

[8] G. A. Elliott and G. Gong, On the classification of $C^{*}$-algebras of real rank zero, II, Ann. Math. 144(1996), 497-610.

[9] R. Exel, Rotation numbers for automorphisms of $C^{*}$-algebras, Pacific J. Math. 127(1987), 31-89.

[10] H. Furstenberg, Strict ergodicity and transformation of the torus, Amer. J. Math. 83(1961), 573-601. 
[11] R. Gjerde and $\varnothing$. Johansen, $C^{*}$-algebras associated to non-homogeneous minimal systems and their K-theory, Math. Scand. 85(1999), 87-104.

[12] T. Giordano, I. F. Putnam, and C. F. Skau, Topological orbit equivalence and $C^{*}$-crossed products, J. reine angew. Math. 469(1995), 51-111.

[13] R. H. Herman, I. F. Putnam, and C. F. Skau, Ordered Bratteli diagrams, dimension groups, and topological dynamics, International J. Math. 3(1992), 827-864.

[14] S. Hu, H. Lin, and Y. Xue, Tracial topological rank of extensions of $C^{*}$-algebras, Math. Scand. 94(2004), 125-147.

[15] B. Itzá-Ortiz, The $C^{*}$-algebras associated to time-t automorphisms of mapping tori, in preparation.

[16] R. Ji, On the Crossed Product $C^{*}$-Algebras Associated with Furstenberg Transformations on Tori, Ph.D. Thesis, State University of New York at Stony Brook, 1986.

[17] K. Kodaka, The positive cones of $K_{0}$-groups of crossed products associated with Furstenberg transformations on the 2-torus, Proc. Edinburgh Math. Soc. 43(2000), 167-175.

[18] J. Kulesza, Zero-dimensional covers of finite dimensional dynamical systems, Ergod. Th. Dynam. Sys. 15(1995), 939-950.

[19] H. Lin, Approximation by normal elements with finite spectra in simple AF-algebras, J. Operator Theory 31(1994), 83-98.

[20] H. Lin, Tracially AF $C^{*}$-algebras, Trans. Amer. Math. Soc. 353(2001), 693-722.

[21] H. Lin, The tracial topological rank of $C^{*}$-algebras, Proc. London Math. Soc. 83(2001), 199234.

[22] H. Lin, Classification of simple $C^{*}$-algebras with tracial topological rank zero, Duke Math. J., to appear.

[23] H. Lin, Simple nuclear $C^{*}$-algebras of tracial topological rank one, preprint.

[24] H. Lin, Classification of homomorphisms and minimal dynamical systems, preprint.

[25] H. Lin and H. Matui, Minimal dynamical systems and approximate conjugacy, preprint (math.OA/0402309).

[26] H. Lin and H. Matui, Minimal dynamical systems on the product of the Cantor set and the circle, preprint.

[27] H. Lin and H. Osaka, The Rokhlin property and the tracial topological rank, J. Funct. Anal., to appear (math.OA/0402094).

[28] Q. Lin, Analytic structure of the transformation group $C^{*}$-algebra associated with minimal dynamical systems, preprint.

[29] Q. Lin and N. C. Phillips, Ordered K-theory for $C^{*}$-algebras of minimal homeomorphisms, pages 289-314 in: Operator Algebras and Operator Theory, L. Ge, etc. (eds.), Contemporary Mathematics vol. 228, 1998.

[30] Q. Lin and N. C. Phillips, Direct limit decomposition for $C^{*}$-algebras of minimal diffeomorphisms, pages 107-133 in: Operator Algebras and Applications, Advanced Studies in Pure Mathematics vol. 38, Mathematical Society of Japan, 2004.

[31] Q. Lin and N. C. Phillips, The structure of $C^{*}$-algebras of minimal diffeomorphisms, in preparation.

[32] J. A. Packer, K-theoretic invariants for $C^{*}$-algebras associated to transformations and induced flows, J. Funct. Anal. 67(1986), 25-59.

[33] N. C. Phillips, When are crossed products by minimal diffeomorphisms isomorphic?, pages 341-364 in: Operator Algebras and Mathematical Physics (Conference Proceedings, Constanţa, (Romania) July 2-7, 2001), J.-M. Combes, J. Cuntz, G. A. Elliott, G. Nenciu, H. Seidentop, Ş. Strătilă (eds.), The Theta Foundation, Bucharest, 2003.

[34] N. C. Phillips, Recursive subhomogeneous algebras, preprint (math.OA/0101156).

[35] N. C. Phillips, Cancellation and stable rank for direct limits of recursive subhomogeneous algebras, preprint (math.OA/0101157).

[36] N. C. Phillips, Real rank and property (SP) for direct limits of recursive subhomogeneous algebras, preprint.

[37] N. C. Phillips, Crossed products by finite cyclic group actions with the tracial Rokhlin property, preprint.

[38] M. Pimsner and D. Voiculescu, Exact sequences for K-groups and Ext-groups of certain cross-products of $C^{*}$-algebras, J. Operator Theory 4(1980), 93-118.

[39] S. Popa, On local finite dimensional approximation of $C^{*}$-algebras, Pacific J. Math. 181(1997), 141-158. 
[40] I. F. Putnam, The $C^{*}$-algebras associated with minimal homeomorphisms of the Cantor set, Pacific J. Math. 136(1989), 329-353.

[41] I. F. Putnam, On the topological stable rank of certain transformation group $C^{*}$-algebras, Ergod. Th. Dynam. Sys. 10(1990), 197-207.

[42] K. Reihani and P. Milnes, $C^{*}$-algebras from Anzai flows and their K-groups, preprint.

[43] M. Rørdam, Classification of certain infinite simple $C^{*}$-algebras, J. Funct. Anal. 131(1995), $415-458$

[44] J. Rosenberg and C. Schochet, The Künneth theorem and the universal coefficient theorem for Kasparov's generalized K-functor, Duke Math. J. 55(1987), 431-474.

[45] J. Tomiyama, Topological full groups and structure of normalizers in transformation group $C^{*}$-algebras, Pacific J. Math. 173(1996), 571-583.

Huaxin Lin

email: hxlin@noether.uoregon.edu

Department of Mathematics

East China Normal University

Shanghai, China

and (current)

Department of Mathematics

University of Oregon

Eugene, Oregon 97403-1222

U.S.A.

N. Christopher Phillips

email: ncp@darkwing.uoregon.edu

Department of Mathematics

University of Oregon

Eugene, Oregon 97403-1222

U.S.A. 